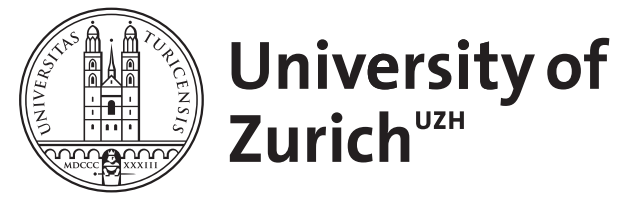

Zurich Open Repository and Archive

University of Zurich

University Library

Strickhofstrasse 39

CH-8057 Zurich

www.zora.uzh.ch

Year: 2009

\title{
Modeling plant morphogenesis and growth
}

Smith, R S

Posted at the Zurich Open Repository and Archive, University of Zurich ZORA URL: https://doi.org/10.5167/uzh-38726

Book Section

Originally published at:

Smith, R S (2009). Modeling plant morphogenesis and growth. In: New Trends in the Physics and Mechanics of Biological Systems. Oxford: Oxford University Press, online. 


\title{
Modeling Plant Morphogenesis and Growth
}

\author{
Richard S. Smith
}

Institute of Plant Sciences, University of Berne, CH-3013 Berne, Switzerland 



\section{Contents}

1 Modeling morphogenesis and growth 1

1.1 Morphogenesis and growth 1

1.2 Dynamic systems with dynamic structure 2

1.3 L-systems, the one dimensional case 2

1.4 Extending L-systems beyond one-dimension 3

2 Descriptive models of growing surfaces $\quad 5$

2.1 Abstracting to two dimensions 5

2.2 Modeling a growing shoot apex $\quad 5$

$\begin{array}{lll}2.3 & \text { Modeling a growing leaf } & 7\end{array}$

2.4 Cells and cell division 8

3 A physically-based model of the shoot apex 11

3.1 Physically-based growth 11

3.2 The mass-spring simulation 12

3.3 Local determination of apex and primordium centers 13

3.4 Calculation of distance on the apex surface 14

3.5 The physics simulation 14

4 Patterning in the shoot apex - phyllotaxis 17

4.1 Phyllotaxis $\quad 17$

4.2 An inhibition model of phyllotaxis 18

4.3 A spacing mechanism based on reaction-diffusion $\quad 19$

4.4 A spacing mechanism based on polar transport 22

4.5 A transport-feedback model of phyllotaxis 25

4.6 Combining molecular and physically-based models 27

5 Leaf venation patterning 30

5.1 Sachs' canalization hypothesis 30

5.2 Combining models on a growing leaf 32

5.3 A dual transport-feedback model for midvein formation 33

5.4 A dual transport-feedback model on a growing cellular template $\quad 35$

6 Conclusion 38

7 Acknowledgments 39

$\begin{array}{ll}\text { References } & 40\end{array}$ 


\section{Modeling morphogenesis and growth}

\subsection{Morphogenesis and growth}

Multicellular organisms develop from embryo to adult through a carefully controlled combination of morphogenesis and growth. Morphogenesis is usually thought of in terms of biochemical compounds, such as gene products and signaling molecules, that give cells the developmental cues required to differentiate and specialize into the various tissues required in the adult organism (Turing, 1952). This idea of differentiation is critical in order to create complex form. If every cell was to do the same thing, then the complex shapes and specialized tissues that we see in the adult forms would not be possible. Since all cells in an organism typically contain the same genetic code, how do they decide to do different things?

Cells separated from two-cell mouse embryos can give rise to two identical adults, but if these same two cells are left together, they are able to coordinate their behavior, each becoming a founder cell for only a portion of the adult. Although this can only be done in animals at very early stages of development, plants are much more flexible, as entire plants can be regenerated from single cells taken from leaves or other tissues. As these single cells divide, how do their progeny collectively decide which ones will become shoot and which ones will become root? Furthermore, if larger pieces of tissue are taken, how is it that these groups of cells are able to organize into a single plant?

This question in morphogenesis recurs repeatedly throughout development. For example, when the plant creates a new leaf, how does it decide which cells will become veins, and which will become intervening tissue? This is a question of pattern formation, and because of their innate flexibility, plants provide an excellent system in which to study this phenomenon. In fact, unlike animals, plants maintain undifferentiated stem-like cells throughout their lifetime at the shoot and root tips, in structures called meristems (Lyndon, 1998). The undifferentiated cells at the tip of the shoot meristem are founder cells from which all of the aerial structures of the plant develop. A similar situation occurs in the root, with root apical meristems providing founder cells for the sub surface portion of the plant.

Another key difference between animals and plants is that plants are sessile and cells cannot move around. Thus the forms we see result from differential and polar growth. After the decision is made to differentiate, cells will become specialized for their new role in the organism, which often involves changes in the biochemical substances that control growth. Thus in order to model many aspects of development, it requires both a biochemical model of the morphogenetic substances superimposed on a model of growth. 


\subsection{Dynamic systems with dynamic structure}

Dynamics systems can be used to model many aspects of development. The chosen domain is characterized by a set of variables that represent the state of the model, with functions to describe their evolution over time. In very simple cases, it will be possible to find analytical solutions for the change in state, or trajectory of the system over time. However this is usually not possible, and numerical simulation on a computer is a more practical alternative. This usually requires the decomposition of the model domain into discrete components to facilitate computation. In some cases this discretization is somewhat arbitrary, such as when using the finite element method to simulate the mechanical deformation of a section of a cell wall under the application of forces. The cell wall is considered as a continuous, although possibly anisotropic, material and apart from numerical error, any discretization should yield the same result. In other cases the domain may have a natural discretization which is not so easily captured with a continuum model. Models of pattern formation often involve cell-to-cell communication, making the discretization into cells a more suitable choice. Likewise, when modeling an entire plant, it might be convenient to discretize at the organ level, with each model component representing a leaf, flower or internode.

Whatever the discretization chosen, developmental models often involve not only changes to the state of the system, but also changes to the underlying structure. Cells may divide into daughter cells, or plants may produce new organs. Models in which both the state and the structure of the model changes over time are called dynamic systems with dynamic structure (Giavitto and Michel, 2001). Although the theory for dynamic systems is well developed, relatively few methods have been proposed for simulating systems with dynamic structure.

\subsection{L-systems, the one dimensional case}

At the level of entire plants, L-systems are particularly convenient for modeling development. Named after Aristid Lindenmayer, they were originally introduced as a formalism to describe the development of filamentous organisms (Lindenmayer, 1968a; Lindenmayer, 1968b). They have since been used to model a wide variety of phenomena that have one-dimensional topology, such as lines of cells or branching structures; anything that can be represented by an acyclic graph. Prusinkiewicz and Lindenmayer (1990) present an excellent introduction to the use of L-system to model the development of plants.

L-systems are rewriting systems based on strings. The strings are made up of modules or letters from a given alphabet. Beginning with an initial string called the axiom, rules are defined that map each module to one or more new modules. The symbols [ ] are used to indicate branches, with the modules contained within considered to be on the branch. Over the time evolution of the simulation, modules are replaced with more complicated groups of modules, causing the structure to grow (Figure 1.1). Parameters can be added to the modules to store data associated with the model component, such as the levels of biochemical substances. Context sensitive rules can be used to simulate inter-module communication.

Note that the L-systems themselves do not specify geometry, only the topology, of the model. However extensions to the theory, such as the Logo-style turtle interpre- 

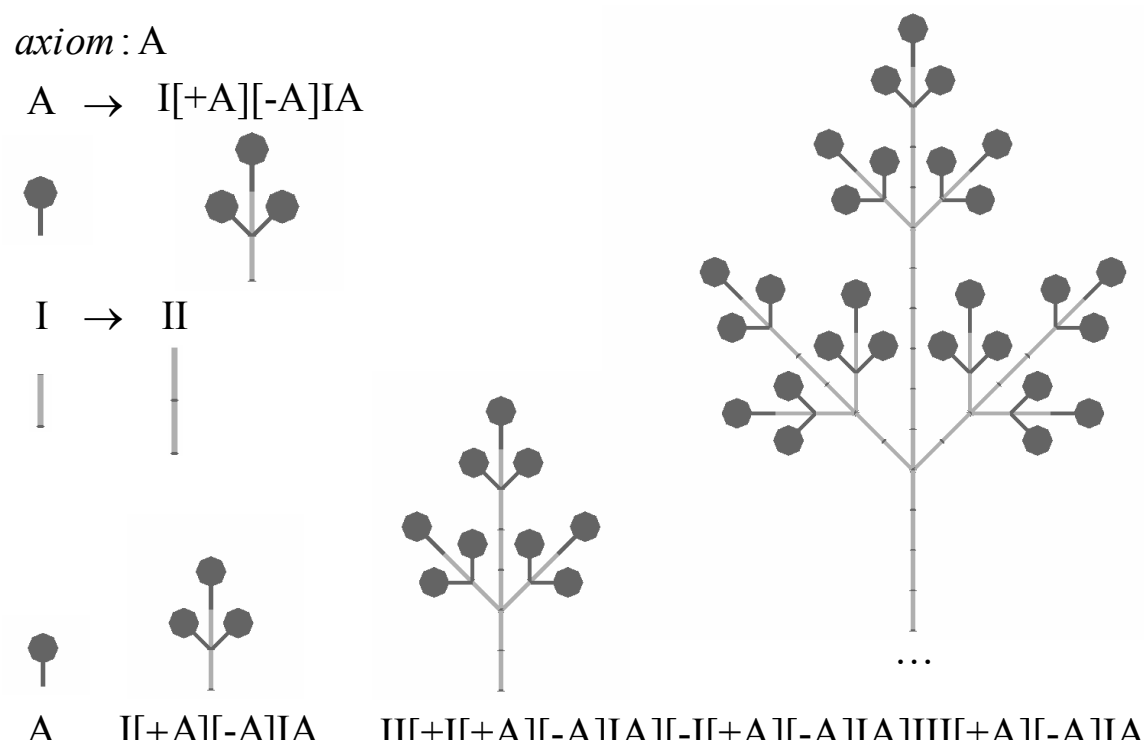

A $\quad$ I[+A][-A]IA $\quad$ II[+I[+A][-A]IA][-I[+A][-A]IA]III[+A][-A]IA

Fig. 1.1 This simple L-system demonstrates how development can be seen as a rewriting process. Modules are are replaced by more modules at each step, causing the structure to grow. Plus and minus signs inside brackets indicate left and right branches. (Adapted from Prusinkiewicz et al. (1996)).

tation of L-systems strings (Prusinkiewicz, 1986) provide a natural way incorporate geometry directly into model. Additional modules which are not model components are added to the string to control the movement of an imaginary turtle. Module parameters are added to control the size of model elements, and to control the orientation between them. Because the underlying topology is that of an acyclic graph, it is always possible for modules to be inserted, deleted, or for the size or orientation between them to change without destroying the overall coherence of the structure (although collisions may occur).

\subsection{Extending L-systems beyond one-dimension}

Severals attempts have been made to extend the notion of L-systems to two or more dimensions. Map L-systems (Lindenmayer and Rozenberg, 1979), cell systems (de Boer et al., 1992), and MGS (Giavitto and Michel, 2001) can all be considered as rewriting systems that operate on graphs rather than strings. Although these formalisms have had some success, it has not proved to be easy to specify update rules on a more general graph structure. In order to uniquely specify context and ensure that neighborhood connectivity remains coherent, update rules can become extremely complex.

An alternative approach was taken by Smith et al. (2004) who introduced vertexvertex systems (VV). VV was originally conceived as a modeling formalism to allow the local specification of mesh subdivision algorithms. The mesh was represented as a 


\section{Modeling morphogenesis and growth}

graph rotation system, a directed graph structure that maintains an ordering of each vertex's neighborhood. In geometric terms, this ordering allows the representation of orientable 2-manifolds (two-dimensional surfaces) embedded in three-dimensional space. Loosely speaking, the fact that the surface is orientable means that it has two sides, which can be distinguished at vertices by examining the ordering of their neighbors. This simple addition to a standard notion of a directed graph turns out to be surprising useful for this class of problems.

Unlike L-systems, however, VV is not implemented as a set of rewriting rules that update the state and structure of the model. Instead, it is implemented as a collection of language statements embedded in $\mathrm{C}^{++}$, although versions now exist based entirely on $\mathrm{C}^{++}$class libraries. Although in some ways $\mathrm{VV}$ is not as elegant as L-systems or MGS, it is practical, and its procedural nature makes it convenient for handling complex models. In addition, the widespread availability of class and template libraries for $\mathrm{C}^{++}$is also a great asset. The simulations presented in the rest of these lecture notes were all programmed using VV under the L-studio (Prusinkiewicz, 2004) modeling environment. 


\section{2 \\ Descriptive models of growing surfaces}

\subsection{Abstracting to two dimensions}

Although the branching structure of plants is innately one-dimensional, the cellular makeup of most plant tissue has a three-dimensional topology. However, it is often possible to abstract the problem to a two-dimensional representation of the tissue. When exploring signaling events that occur primarily in a relatively thin layer of cells, it may be possible to abstract this layer to a single layer of cells. This approach has been used for the cambium layer in trees (Kramer, 2002), the surface layer of cells in the shoot apex (Jönsson et al., 2006; Smith et al., 2006; Barbier de Reuille et al., 2006), as well as the mesophyll layer in leaves (Mitchison, 1980; Kramer, 2004; Rolland-Lagan and Prusinkiewicz, 2005; Barbier de Reuille et al., 2007; Stoma et al., 2008). Two dimensional geometries may also be obtained by using a cross sectional representation of the tissue. This has been proposed for both the shoot (Stoma et al., 2008; Bayer et al., 2009) and root (Grieneisen et al., 2007; Stoma et al., 2008) apices. Most cellular level simulation models of plant morphogenesis and patterning use this simplification.

\subsection{Modeling a growing shoot apex}

The plant shoot apex consists an external layer of cells, called the tunica, that surrounds the inner tissue known as the corpus. The molecular processes leading to the positioning of new organs, which results in phyllotaxis patterning, are thought to occur in this outer layer(Reinhardt et al., 2003; Smith et al., 2006). Thus it is sufficient to consider only the surface layer for simulation purposes.

This surface layer can be modeled as a surface of revolution, generated by rotating a planar curve around the longitudinal axis of the apex (Figure 2.1). This planar curve is a B-spline (Foley et al., 1990), defined interactively using a graphical editor available within L-studio, and can easily be changed to model apices with various profiles. A point on the apex surface is thus characterized by two coordinates $(\theta, a)$ where $\theta$ is the angle of rotation around the axis of the apex, measured with respect to a reference direction, and $a$ is the distance from the apex tip, measured along the generating curve on the apex surface. In Cartesian coordinates, the $(x, y, z)$ position of a surface point $S(\theta, a)$ is thus given by:

$$
S(\theta, a)=(x(a) \cos (\theta), y(a), x(a) \sin (\theta)) .
$$




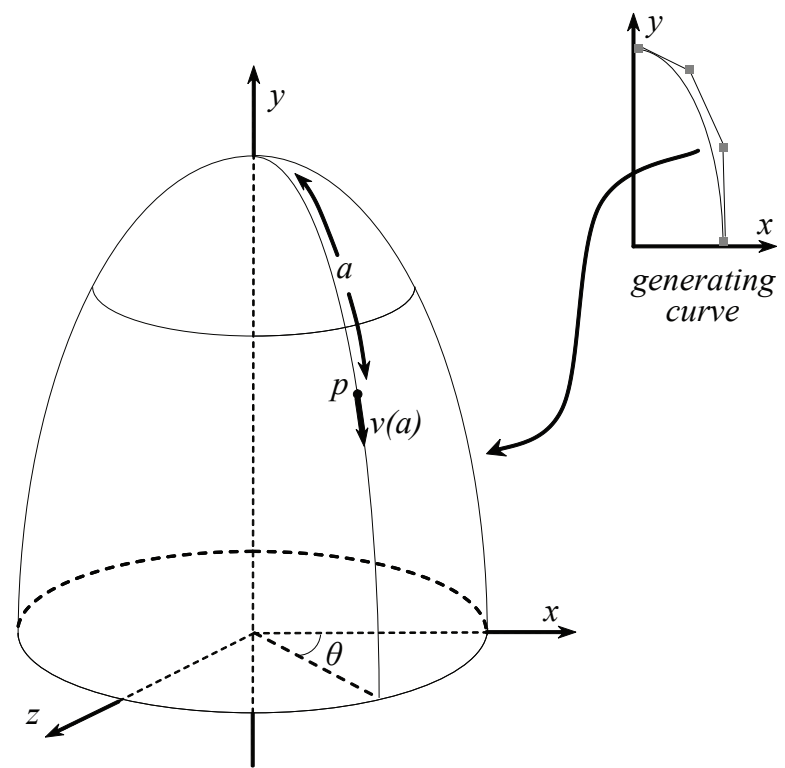

Fig. 2.1 A two-dimensional representation of the shoot apex. The apex shape is defined by a B-spline generating curve rotated around the longitudinal axis of the shoot apex. A sample point $P$ on the surface with coordinates $(\theta, a)$ moves away from the apex tip with the velocity $v(a)$.
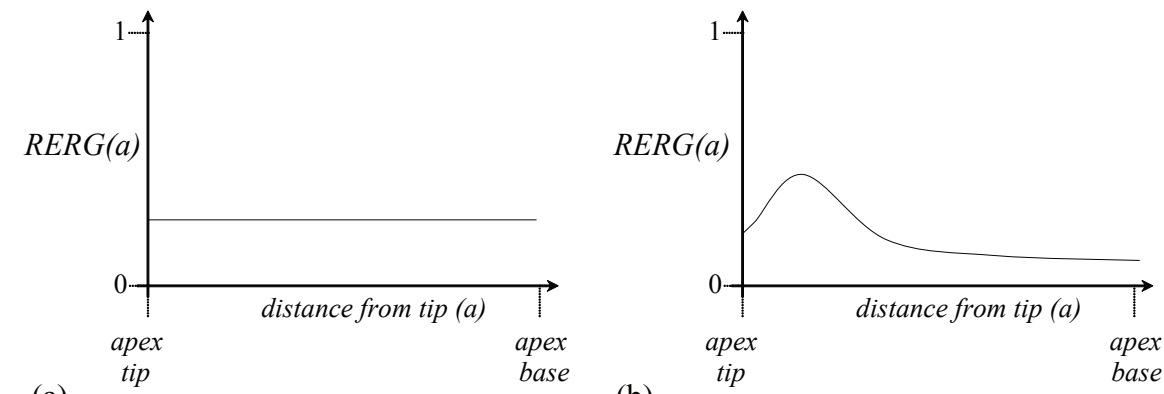

(b)

Fig. 2.2 Sample plots defining relative elemental rate of growth $R E R G$ as functions of distance a from the apex tip. The functions are defined graphically, using an interactive B-spline editor. (a) Constant growth function. (b) Function with a decreased growth rate at the apex tip and flank. 


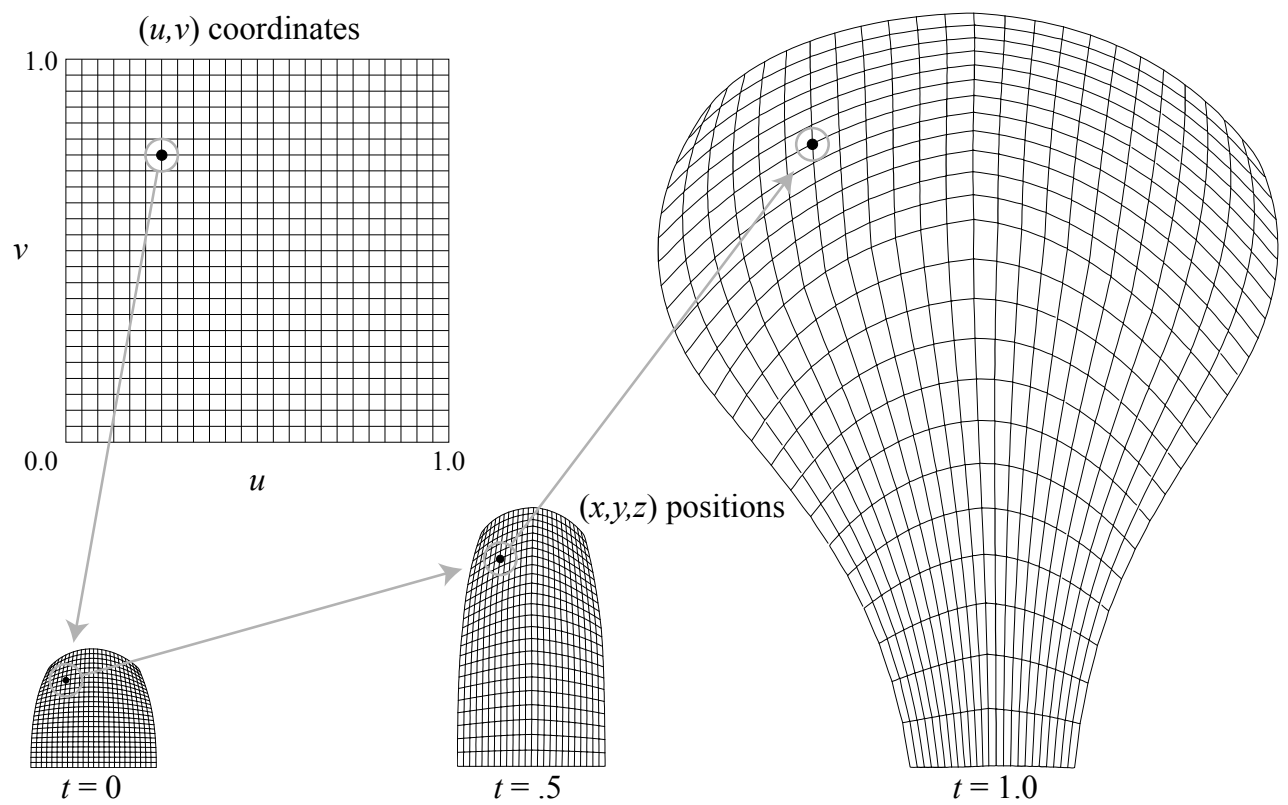

Fig. 2.3 Modeling a growing leaf by using Bezier surfaces. A sequence of Bezier surfaces represents the leaf at various times $t$ during the simulation. Coordinates in the parameter space $(u, v)$ shown in the top left are mapped into three-dimensional space $(x, y, z)$ as shown. The position of points at time steps in between the three key frames is found by linear interpolation.

Growth is simulated by moving points away from the apex tip while preserving the overall shape of the reference surface. This motion is characterized by a function $R E R G(a)$, which defines the relative elemental rate of growth (Hejnowicz et al., 1984) in the longitudinal direction (along the generating curve) at a distance $a$ from the apex tip (Hejnowicz et al., 1984; Nakielski, 2000). The velocity with which a point $S(\theta, a)$ moves away from the apex tip along the generating curve is then given by the integral:

$$
v(a)=\int_{0}^{a} \operatorname{RERG}(a) d a .
$$

Similar to the generating curve, the growth function $R E R G(a)$ can be defined graphically within L-studio, which makes it easy to specify various distributions of growth rates on the apex surface. It is often the case that growth is slower at the very tip of the apex in the stem cell niche (Figure 2.2). To speed computation, a lookup table for the values of the integral given in Equation 2.2 can be precomputed at startup.

\subsection{Modeling a growing leaf}

The growing apex model in the previous section is suitable for modeling radially symmetric surfaces that grow at the tip, such as the shoot and root apices. Surfaces such 

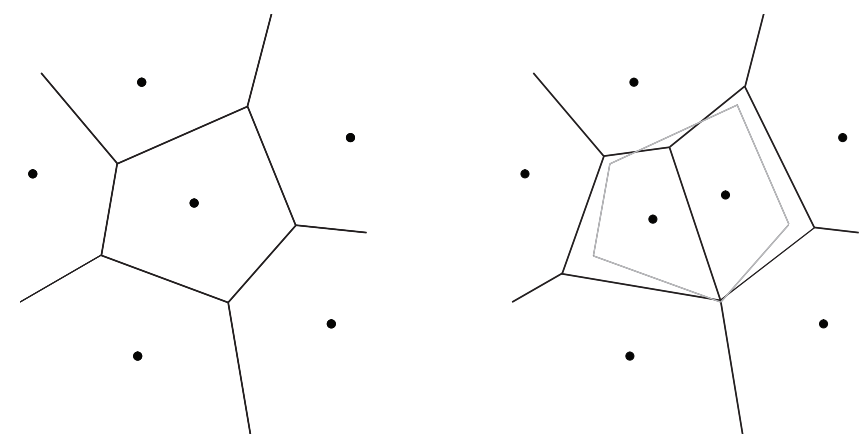

Fig. 2.4 Cell division in a tissue with cells represented by Voronoi regions. Unrealistic changes in the cells' geometry are introduced during cell division. Cell before division (left), and adjusted regions after division (right). Note the shortening and lengthening of some of the neighbor cell walls as wall junctions appear to "move".

as leaves do not show this symmetry and have growth that is distributed throughout the surface. In this case it is possible to model the surface using a key framing technique often used in computer graphics. The shapes of several stages of the growing leaf are specified by defining a sequence of Bezier surfaces that represent the leaf at different times (key frames) during the simulation (Figure 2.3). These surfaces can be defined interactively using the surface editor provided with L-studio. Interpolation of the control points of the surfaces is used to produce the leaf at any desired point between the key frames. By advancing time in small steps, a smoothly growing leaf surface can be produced.

The Bezier surfaces used to model the leaf are two-dimensional parametric surfaces embedded in three-dimensional space. Points on the surface are represented by coordinates $(u, v)$ which are mapped by Bezier surface evaluators (Neider et al., 1994) to positions $(x, y, z)$. As the simulation progresses and the leaf changes shape, a $(u, v)$ coordinate at the tip of the leaf will remain at the tip even though its actual position in space might change considerably. A surface point $S(u, v)$ is computed using the control points $\left\{P_{i j}\right\}$ as follows:

$$
S(u, v)=\sum_{i=0}^{n} \sum_{j=0}^{m}\left(\begin{array}{c}
n \\
i
\end{array}\right) u^{i}(1-u)^{n-i}\left(\begin{array}{c}
m \\
j
\end{array}\right) v^{j}(1-v)^{m-j} P_{i j}
$$

The binomial coefficients in the above expression can be precomputed once at startup to speed the calculation.

\subsection{Cells and cell division}

Most static models of morphogenesis and patterning in plants have been implemented by using rectangular grids of cells. However when considering growing, and possibly irregularly shaped surfaces, rectangular cells are no longer practical. One possibility is to use Voronoi diagrams to model cells (Honda, 1978; Jönsson et al., 2006) because of 
(a)

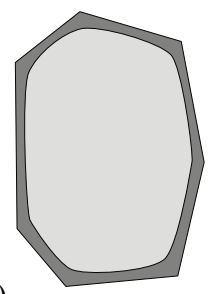

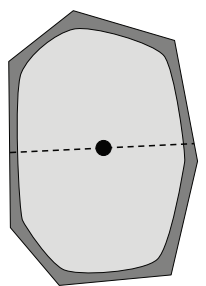

(b)

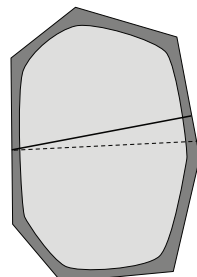

(c)

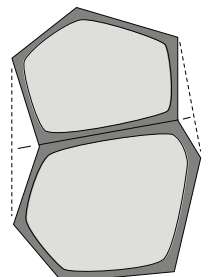

(d)

Fig. 2.5 Model of cell division. (a) A mother cell before division. (b) The chosen dividing wall is the shortest wall passing through the center of the mother cell. (c) The endpoints of the wall are displaced from any preexisting vertices of the mother cell in order to avoid 4-way junctions. (d) The dividing wall is shortened. (Adapted from Smith et al. (2006)).

their irregular cell-like appearance. They are not ideal for modeling growing plant tissues, however, since the rearrangement of Voronoi regions that inevitably occurs during cell division creates unrealistic motions of cell walls (Figure 2.4). Such motions are not possible in plants due to the rigidity of the interconnected cell walls that surround plant cells. More realistic divisions can be obtained by using map L-systems (Lindenmayer and Rozenberg, 1979; Prusinkiewicz and Lindenmayer, 1990) and cell systems (de Boer et al., 1992). However, these systems rely on physically-based mass-spring simulations to produce realistically shaped cells, and are thus not directly applicable to the purely descriptive growth model described in Sections 2.2 and 2.3.

A model for cell division proposed by Nakielski (2000) for the shoot apex can be generalized to much wider class of growing two dimnesional surfaces, including those described above (Smith et al., 2006; Smith and Bayer, 2009). Cells are modeled as polygons, with the position of cell vertices changing over time as a result of surface growth. Cell division occurs when the cell size (polygon area) reaches a threshold value. Inspired by Errera's rules for cell divsion (Errera, 1888), the shortest wall through the center of the cell is chosen for division. The position of the dividing wall may be adjusted to avoid four-way junctions, which are unusual in plant tissue. To produce more realistic cell shapes, the newly divided cell is "pinched" slightly by moving the vertices of the dividing wall slightly towards each other (Figure 2.5). These rules for cell division result in cellular patterns similar to those in observed in plant tissue, and over a wide range of surface shapes and growth functions (Figure 2.6).

Cell division requires the insertion of new vertices into the growing surface, as new wall junction vertices are created. Equations 2.1 and 2.3 are used to calculate the position in space of a surface point from an arclength-angle pair in the case of the apex surface, or a pair of parametric coordinates in the case of the Bezier surface model. However, they do not lead easily to closed formula to compute the reverse operation. In fact, the points of new vertices created during cell division in general will not lie on the surface, and instead the closest point on the surface must be found. This can be done by applying a Newton-Raphson root search technique to equations 2.1 and 2.3 for each new point inserted into the surface. 
10 Descriptive models of growing surfaces
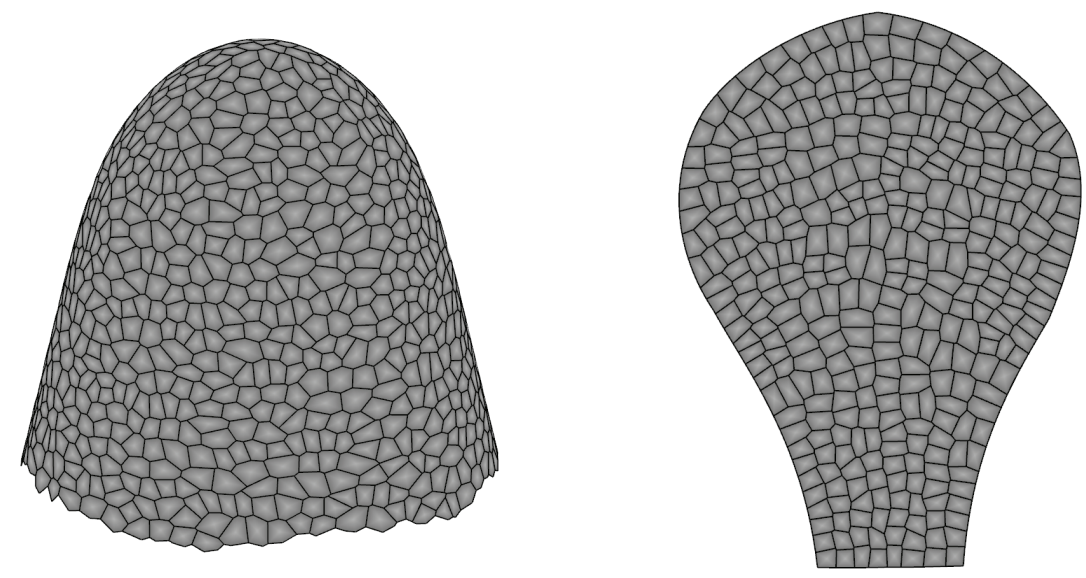

Fig. 2.6 Cell patterns on growing surfaces. Cell division with the "shortest wall through center" rule creates cellular patterns similar to plant tissue. 


\section{3}

\section{A physically-based model of the shoot apex}

\subsection{Physically-based growth}

In the growing surface models discussed in the previous sections, the shape of the surface was provided as an input to the models. With physically-based models, the shape can be an emergent property of the model. This opens up the possibility of modeling the processes that lead to these emergent shapes, and the interaction between morphogens, growth, and form. Since it is also widely believed that mechanics might play a direct role in morphogenesis, physically-based models are becoming increasingly important. For a review of the effects of mechanical forces on plant morphogenesis see Dumais (2007).

Several types of physically-based models have been proposed for the shoot apex, and most are based on mass-spring systems. Although (Hamant et al., 2008) have presented a finite element model for the shoot apex, this model does not include growth. Finite element models have advantages over mass-spring systems as they have a direct connection with continuum mechanics, allowing parameters such as Young's modulus and Piosson ration to be used directly in the model. It is not clear how to translate these parameters into equivalent spring constants, especially for elements with dimension greater than one. Nevertheless, mass-spring systems are able to model very complex behavior, often in real time, and are relatively easy to program (see Figure 3.1).

In the simplest case, a surface is created by using vertices that represent only the cell centers connected by a network of springs (Jönsson et al., 2006). If required, the cell walls can be calculated by the finding the Voronoi regions of the cell centers, or the cells can simply be rendered as intersecting spheres. Alternatively, the cells can be defined by their walls as in the models described sections 2.2 and 2.3. In order to prevent the surface mesh from collapsing, a force normal to the surface in the outward direction is applied to each vertex. This force can be viewed as representing the combined effects of turgor pressure and other forces from the cells inside the shoot apex. Since the surface layer of the shoot apex is much stiffer than the inner tissue, the structure can be likened to the skin of a balloon that is holding back the pressure within. In order to anchor the apex in space, the positions of the vertices at the bottom of the apex are fixed. Growth can be simulated by increasing the rest lengths of the springs, by subdividing and adding additional springs, or by a combination of these methods. 

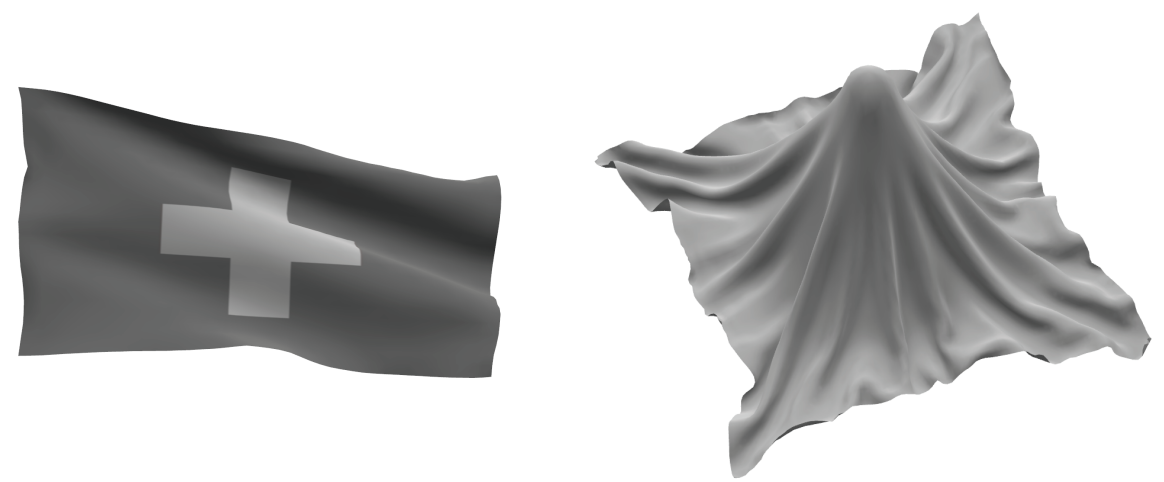

Fig. 3.1 Examples of two-dimensional surface simulations using mass-spring systems. Flag blowing in the wind (left) and a wrinkling cloth (right). Realistic looking shapes can be created with very simple programs, often in real time.

This type of model makes is possible to consider locally induced differences in growth rate, a property that is difficult to achieve in purely descriptive models. Biochemical substances can be used to control the local rate of increase in the rest lengths of the springs, simulating the actions of wall relaxing enzymes thought to cause growth. Modeling the outgrowth of organs from the surface is straightforward, and it is possible to model shapes that are difficult to define descriptively.

\subsection{The mass-spring simulation}

The mass-spring model of the shoot apex presented here replaces the descriptive surface growth model described in sections 2.2 and 2.3 with a simple physics simulation. The cell walls are considered to be springs that are connected to point masses located at the junctions between walls. The point masses at all such junctions are considered to have an identical value in abstract units of 1 . The force acting on the point mass of a vertex $v$ located at position $p_{v}$ due to springs $\mathbf{F}_{s_{v}}$ is based on Hooke's law and is calculated as:

$$
\mathbf{F}_{s_{v}}=\sum_{u \in N_{v}} k\left(1-\frac{\left\|\mathbf{p}_{u}-\mathbf{p}_{v}\right\|}{l_{v \rightarrow u}}\right) \frac{\mathbf{p}_{u}-\mathbf{p}_{v}}{\left\|\mathbf{p}_{u}-\mathbf{p}_{v}\right\|}
$$

where $N_{v}$ is the neighbors of vertex $v, \mathbf{p}_{u}$ is the position of a neighbor vertex $u, k$ is the spring constant per unit length, and $l_{v \rightarrow u}$ is the rest length of spring joining vertex $v$ to neighbor neighbor vertex $u$. The norm symbol indicates the Euclidean norm or distance between the points. The expression within the brackets is positive when the distance between $v$ and its neighbor $u$ less than the rest length, and negative if it is greater. Note that for a given difference from the rest length, the magnitude of the force is reduced as the rest length of the spring increases. This magnitude is then multiplied by the normalized vector $\left(\mathbf{p}_{u}-\mathbf{p}_{v}\right) /\left\|\mathbf{p}_{u}-\mathbf{p}_{v}\right\|$ and by $k$ to give the forces. These forces are then summed over all the neighbors of the vertex and multiplied by the spring constant which corresponds to the stiffness of the springs. 
In addition to the forces on a vertex due to springs, a uniform force representing the internal turgor pressure $\mathbf{F}_{t}$ is included in the model which acts in the direction of the surface normal at each vertex. A real plant apex is able to control its growth direction, enabling a shoot to grow straight, to favor an upward growth direction, or to grow towards the light. These processes are not modeled directly but are accounted for by a directional force $\mathbf{F}_{d}$ which is applied to the vertices at the tip of the apex where directional growth occurs. This directional force is also applied at the tips of growing primordia, although the strength of the force may be different than that used for the main apex. Combining the various components, the total force from all sources $\mathbf{F}_{a_{v}}$ acting on a vertex $v$ is:

$$
\mathbf{F}_{a_{v}}=\mathbf{F}_{s_{v}}+\mathbf{F}_{t_{v}}+\mathbf{F}_{d_{v}}
$$

Note that for cells in the apex which are not at the tip of a growing apex or primordia, the last term $\mathbf{F}_{d_{v}}$ is zero.

Once the forces on the vertices are calculated, the steady-state of the system is determined (see Section 3.5), and growth is simulated by increasing the rest lengths of the springs. If a spring is longer than its rest length, then its rest length is increased proportional to this difference. In the case of cell walls within the extent of an apex or primordium tip, this increase is larger, and the directional force $\mathbf{F}_{d}$ is also applied. This causes the apex and bulging primordia to grow quickly at the tips, while still increasing in size at a lesser rate at locations more distal from the tips.

\subsection{Local determination of apex and primordium centers}

In the previous section, forces and increases in spring rest length were calculated differently for vertices near the tip of the apex. In general it is not straightforward to determine the center of the apex tip, and which cells belong to it, in cellular models with emergent growth. The problem is even more difficult when trying to determine the centers of newly protruding leaf or flower primordia. In models on regular grids a single vertex can be designated as the center of the tip of the apex or primordium if the subdivision scheme used keeps this vertex at the center as the simulation proceeds. In cellular models, however, a central cell may divide, causing the center of the tip of the apex or primordium to shift as one of the new daughter cells is chosen as the center. In addition, the irregularity of the wall lengths can cause cells to grow at different rates, shifting the location of a central cell of an apex or primordium tip.

To overcome this problem, a method is needed so that the position of an apex or primordium center can be determined on the basis of local information contained in the cells that make up the primordium itself. Ideally, this would involve modeling the molecular components that are responsible for determining apex or primordium identity, extent, and boundary. In the model presented here, a somewhat simpler approach is taken which nonetheless retains a local character.

For the main apex, the center is specified at the start of the simulation, whereas primordium centers are determined as the simulation proceeds. Along with the location of the center, an initial extent of the apex or primordium is specified, which may change as the apex or primordium ages. All of the vertices within the radius of this initial extent are considered as part of the growing tip. These vertices are subject to 
the directional force $\mathbf{F}_{d}$ discussed previously, and participate in the dynamic process of determining the center of the tip. At the end of each time step, after growth, the physics simulation, and any cell divisions have occurred, all of the positions of the vertices belonging to the apex or primordium center from the previous step are averaged to determine a tentative center point for the next iteration. This average is weighted in favor of vertices closer to the previous center, and is in general not on the apex or primordium surface. A point on the surface is obtained by projecting this tentative center onto the surface layer of cells, in the direction of force $\mathbf{F}_{d}$. All of the points that are within the apex or primordium extent are then updated with the distance to the new center, and will participate in determination of the center at next time step.

\subsection{Calculation of distance on the apex surface}

The maintenance of the apex and primordium centers, as well as the determination of which cells belong to a particular apex or primordium, requires the calculation of their distances to their respective centers. For the main apex itself, Euclidean distance can be used, however this can cause problems with protruding primordia. If the extent of a primordium at initiation reaches to the other side of a tall slender apex, vertices on the other side of the apex might be included. If the tip of one primordium comes too close to the flank of another, it can recruit vertices belonging to a completely different primordium.

This problem is solved by using the distance along the apex surface, instead of the distance through space, when determining how far a vertex is from an apex center. Although calculating the geodesic would give the most accurate measure of distance on the surface, the weighted graph distance (using the lengths of the edges), which can be calculated with Dijkstra's algorithm, has several advantages. Aside from being simple, fast, and easy to implement, graph distance might actually be a more accurate measure of distance as far as the diffusion and transport of molecular components is concerned. This is especially true if it is calculated using a separate graph containing only the centers of the cells.

\subsection{The physics simulation}

In mass-spring simulations it is often desirable to visualize the dynamic behavior of a system as it progresses towards a steady state. This would be the case for the simulation of a bouncing ball, where the dynamic behavior of the physics simulation is of interest. In the case of the growing apex model described here, each time step starts with the simulation at equilibrium. Growth, which changes the rest lengths of the springs, upsets this equilibrium and the system must be solved for the equilibrium state under these new conditions. This means that in general, there will be many iterations of the physical simulation for each growth step of the model.

There are several methods by which the equilibrium state of the system can be determined. Given the total force $\mathbf{F}_{a_{w}}$ on a vertex $w$ as defined earlier, the following equations describe the change in velocity $\mathbf{v}$ and position $\mathbf{p}$ over time: 


$$
\begin{aligned}
\frac{d \mathbf{v}_{w}}{d t} & =\left(\mathbf{F}_{a_{w}}-\varsigma \mathbf{v}_{w}\right) d t \\
\frac{d \mathbf{p}_{w}}{d t} & =\mathbf{v}_{w} d t
\end{aligned}
$$

where $\varsigma$ is the damping constant.

This assumes that the point masses at all of the vertices in the model are equal to 1. Using the forward Euler method, the values for velocity and position at time $t+\Delta t$ can be calculated as:

$$
\begin{aligned}
& \mathbf{v}_{t+\Delta t}=\mathbf{v}_{t}+\left(\mathbf{F}_{a}-\varsigma \mathbf{v}_{t}\right) \Delta t \\
& \mathbf{p}_{t+\Delta t}=\mathbf{p}_{t}+\mathbf{v}_{t} \Delta t
\end{aligned}
$$

Although these formulas give acceptable results, if the velocity at time $t+\Delta t$ is substituted in Equation (3.6) for $\mathbf{v}_{t}$, then the step size can be increased by a factor of five or more in many cases. This method has been termed the symplectic forward Euler method (Stern and Desbrun, 2006). With this modification the update formulas become:

$$
\begin{aligned}
& \mathbf{v}_{t+\Delta t}=\mathbf{v}_{t}+\left(\mathbf{F}_{a}-\varsigma \mathbf{v}_{t}\right) \Delta t \\
& \mathbf{p}_{t+\Delta t}=\mathbf{p}_{t}+\mathbf{v}_{t+\Delta t} \Delta t
\end{aligned}
$$

The physics simulation in each growth step is iterated until an equilibrium state is reached, which is determined by examining the maximum total force acting on any vertex. Since the total force will go to zero when the system is at a steady-state, this can be used to apply a threshold to stop the iteration. In practice it is convenient to also limit the number of iterations, as this improves performance and smooths out the simulation when primordia first appear and tend to "pop out" from the apex surface.

Since only the equilibrium state of the physics simulation is sought at each time step of growth, a relaxation method can be used that does not consider velocity. Vertices can simply be moved in the direction of the total force by some amount proportional to the magnitude of the force. The system is then iterated until the forces approach zero. Both methods produce similar results with similar execution times.

Simulations using a physically-based growth model will in general require considerably more computation time than the simulations using descriptive growth. This is because each growth step requires that the physics simulation be iterated until equilibrium is reached. Despite this drawback, physically-based models of the shoot apex have advantages. Physically-based models can be used to explore how biochemical signals interact with mechanics to produce the emergent shapes that we see as the plant develops. It is possible to explore the effects of mechanics on morphogenesis, as stress information is also available in the model. 
16 A physically-based model of the shoot apex

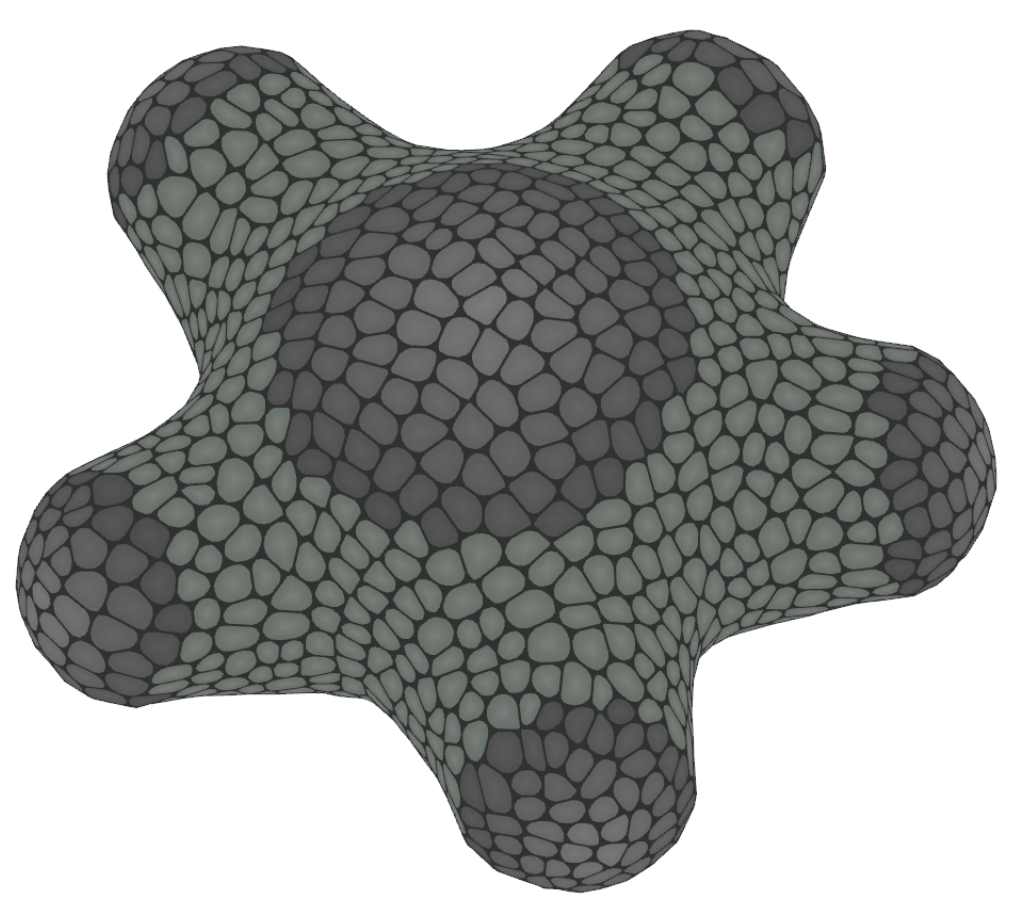

Fig. 3.2 Physically-based model of a hypothetical flower apex with five bulging primordia. Darker cells are considered part of the growing tips of the apex and primordia. Locations of bulging primordia specified as model parameters. 


\section{4 \\ Patterning in the shoot apex - phyllotaxis}

\subsection{Phyllotaxis}

Few patterns in nature are more conspicuous than the intersecting spirals seen in the head of a sunflower, or on the side of a pine cone. In order to create these patterns, the plant must be able to position organs very precisely, sometimes to within a fraction of a degree. How is the plant able to accomplish this?

To examine this question we need to look at the shoot apex, at the tip of a growing plant shoot, for this is where new plant organs are formed. The central zone of the shoot apex consists of undifferentiated founder cells, surrounded by a relatively narrow band

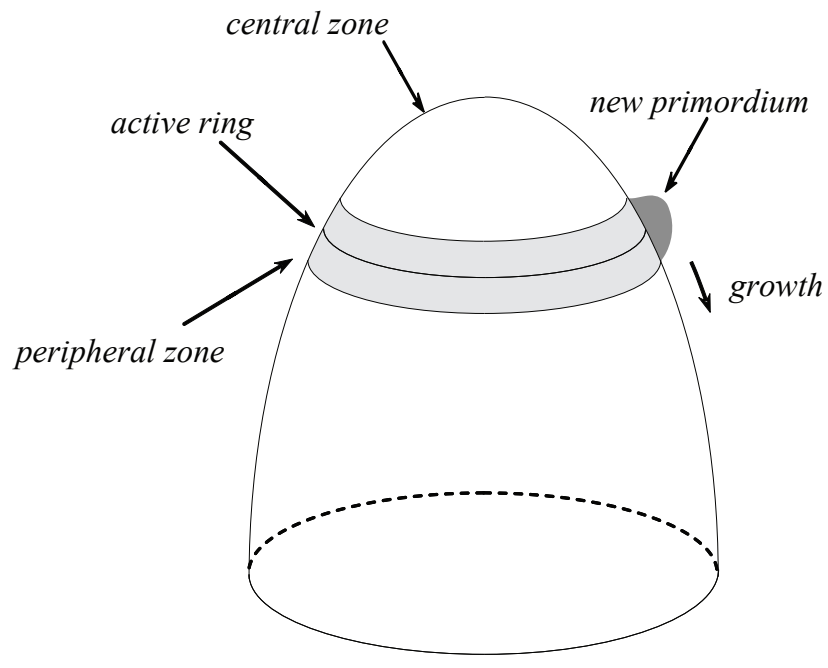

Fig. 4.1 Structure of the shoot apex. The central zone at the tip of the apex contains the stem cell niche, and is surrounded by a narrow band of cells called the peripheral zone, where new organ primordia initiate. In some cases this band of cells is idealized as an active ring. Growth causes the new organs to move down the flank of the apex. 
of cells called the peripheral zone. As the plant develops, the peripheral zone maintains an approximately constant distance from the tip of the apex. Only cells within the peripheral zone are competent to initiate organs. In some cases it is convenient to use the notion of an active ring as an abstraction of the peripheral zone. This is defined to be the line on the apex surface that encircles the apex and is located at the center of the peripheral zone (Figure 4.1). Since the radial position of plant organs changes little after initiation, it is in the shoot apex that the phyllotaxis patterns are formed. The radial position, and timing of initiation of new leaves or florets, determines the macroscopic patterns we see.

A simple observation from Hofmeister (1868) gives insight into what determines the site of a new primordium. He proposed that new plant organs initiate as far as possible from preexisting ones. Thus older primordia somehow inhibit the formation of new ones nearby. Several theories have been suggested for how this inhibition may occur. Existing primordia might release some sort of chemical inhibitor (Schoute, 1913), or perhaps physical forces play a role (Green et al., 1996). Whatever the mechanism for this inhibition, phyllotaxis results from the positional information coming from existing plant organ primordia combined with growth of the shoot tip. Thus in order to study phyllotaxis through simulation, a model of this inhibition, and dynamic model of a growing shoot apex are required.

\subsection{An inhibition model of phyllotaxis}

Can a simple spacing mechanism lead to the complex intersecting spiral patterns we see in plants? Although it is likely possible to treat this problem analytically, it can be easily addressed with computer simulation. Given the growing shoot apex structure as described in Section 2.2, the peripheral zone is modeled as a ring that is maintained a fixed distance from the tip (Figure 4.1).

Simulation of phyllotaxis proceeds in a sequence of time steps. At each step, points on the apical surface, including existing primordia, are moved away from the apex tip according to their velocities (Equation 2.2). The active ring is divided into equally spaced sampling points, and the inhibition from previous primordia is calculated. If the one of these points drops below a given inhibition threshold, a new primordium is placed at the sampling point. In subsequent simulation steps, the new and previous primordia move away from the active ring as a result of the apex growth. This movement, combined with a possible decrease of the inhibiting influences of primordia with their age, reduces the inhibiting strength on the active ring. Over time, this inhibition drops sufficiently at some location to allow for the formation of another primordium, and the process repeats.

It seems biologically plausible that the inhibition from previous primordia would depend inversely on their distance from an active ring sampling point, and that inhibition would decay over time. This leads to the following function to calculate inhibition (?). In a system containing $n$ primordia, the combined inhibiting effect $h$ on a sampling point $S$ is calculated as the sum:

$$
h(S)=\sum_{i=1}^{n} \frac{1}{d\left(P_{i}, S\right)} e^{-b t_{i}}
$$


(a)
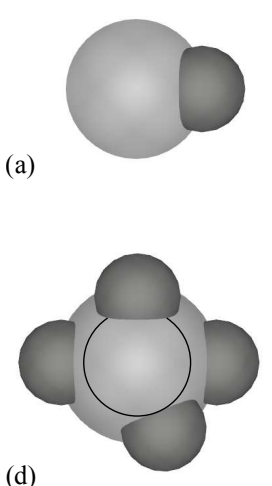

(b)
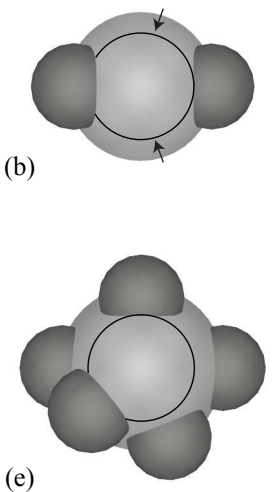

(c)

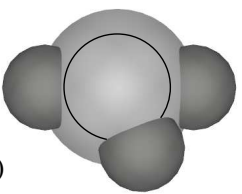

(f)

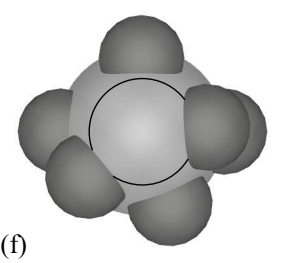

(g)

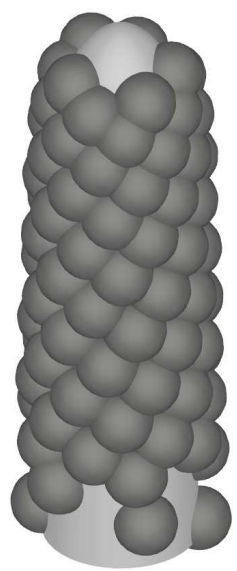

Fig. 4.2 Self-starting spiral Fibonacci phyllotaxis pattern created by using an active ring with 100 sampling points. (a) The initial primordium is arbitrarily placed by the model. (b) The second primordium appears in the area of least inhibition, at a divergence angle of $180^{\circ}$ from the first. At this point there are two minima of inhibition on the active ring, both of which are closer to the older of the two existing primordia (arrows). (c) The third primordium appears at one of these minima, the choice determining the direction of the spiral. (d-f) Successive primordia appear, the position of each being determined by the inhibition from the previous few. This results in a spiral pattern. (g) Once the pattern is established, the inhibition threshold is raised, leading to a more densely packed pattern. Note the rows of spirals in alternating directions. (Adapted from Smith2006a).

where $d\left(P_{i}, S\right)$ is the distance between primordium $P_{i}$ and a sampling point $S$ on the apex surface, $t_{i}$ is the age of primordium $i$, and $b$ controls the rate of exponential decrease in inhibition over time. Initially, when the model is first started, all of the active ring sampling points have zero inhibition, and one point is selected for the first primordium. Figure 4.2 shows how this simple spacing mechanism results in spiral phyllotaxis.

\subsection{A spacing mechanism based on reaction-diffusion}

Since phyllotaxis is the result of the superimposition of a simple spacing mechanism on a growing shoot apex, it is natural to ask how this spacing might occur in the plant in mechanistic terms. This leads back to the initial question of how some cells are selected for differentiation over others, even though they all operate with the same rules or genetic code. This question was addressed by Turing (1952) and his reaction-diffusion model has since become one of the most widely used models for pattern formation in biology (Meinhardt 1982; Murray 2002). Turing showed, using both analytical and computer simulation methods, that a pattern of peaks in concentration of two or more chemical substances, which he termed morphogens, could arise spontaneously in a 


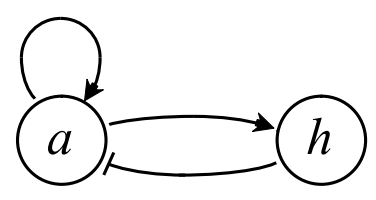

Fig. 4.3 Activator-inhibitor network. The activator $a$ enhances its own production, as well as the production of an inhibitor $h$. The inhibitor suppresses the activator.
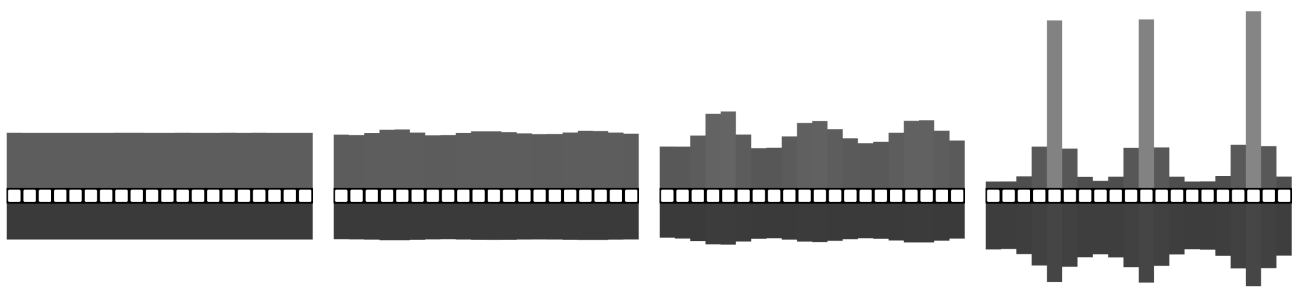

Fig. 4.4 Reaction-diffusion patterning using an activator-inhibitor system on a line of cells. The ends of the line are connected to each other to form a ring. The activator concentration is represented by the height of the bars on top, with the inhibitor below. The model starts with homogenous initial conditions, with a small amount of noise added to the initial activator concentration. This noise destabilizes the system and the activator-inhibitor model produces relatively evenly spaced peaks of activator concentration. Note that both the activator and inhibitor concentrations are high in the activated cells.

ring of cells. Starting with homogenous initial conditions, and using the same rules for changing morphogen concentration in each cell, slight perturbations in the initial conditions due to noise could upset an unstable equilibrium and lead to patterning.

To understand this process, it is instructive to look at a reaction-diffusion system proposed by Gierer and Meinhardt (1972). This model is based on two substances and is called an activator-inhibitor system, with equations that are more intuitive than those proposed by Turing. One substance, called the activator $a$ enhances its own production, as well as that of another substance $h$, termed the inhibitor. The inhibitor inhibits production of the activator. Such a system is easy to envision as a feedback loop in a genetic regulatory network (see Figure 4.3). On a ring of cells, the change in concentration of the activator and inhibitor for each cell are given by:

$$
\begin{gathered}
\frac{d a_{i}}{d t}=\rho_{a_{0}}+\rho_{a} \frac{a_{i}^{2}}{1+h_{i}}-\mu_{a} a_{i}-D_{a} \sum_{j \in N_{i}}\left(a_{i}-a_{j}\right) \\
\frac{d h_{i}}{d t}=\rho_{h_{0}}+\rho_{h} a_{i}^{2}-\mu_{h} a_{i}-D_{h} \sum_{j \in N_{i}}\left(h_{i}-h_{j}\right)
\end{gathered}
$$

where $a_{i}$ and $h_{i}$ is the concentration of activator and inhibitor in cell $i, \rho_{a_{0}}$ and $\rho_{h_{0}}$ control background production (small amounts), $\rho_{a}$ and $\rho_{h}$ control activator-enhanced 

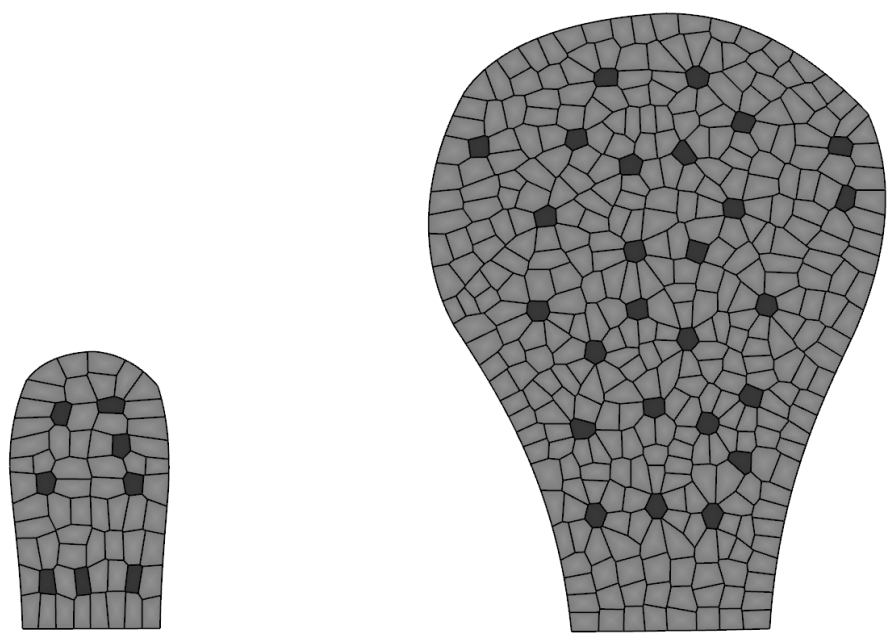

Fig. 4.5 Reaction-diffusion patterning on a cellular model of a growing leaf. The darker cells represent peaks in the activator concentration. As the leaf grows, space opens up in the leaf allowing more peaks to form.

production, $\mu_{a}$ and $\mu_{h}$ control decay, $D_{a}$ and $D_{h}$ control the rates of diffusion cell to cell, with the summations taken over all of the neighbors of cell $i$. Each cell stores a single value for the concentration of activator and inhibitor, and the junction between cells is abstracted to a single interface. For stable peaks to form, the rate of activator diffusion must be much smaller than that of the inhibitor.

Small local maxima in activator concentration due to random variation lead to a local increase in production of both the activator and the inhibitor. The inhibitor diffuses away more quickly than the activator, reducing its effect on local activator self-enhancement, while suppressing activator self-enhancement nearby. In a system of identical cells, each operating with identical rules, this destabilization can lead to a spatial pattern of peaks in activator concentration (Figure 4.4), which can trigger selective differentiation leading to patterning. By changing parameters in Equations 4.2 and 4.3 , it is possible to vary the distance between peaks. The interaction of multiple substances, combined with multiple cascading interactions, has been used to account for a wide variety of patterning processes observed in nature, including phyllotaxis (Meinhardt, 1982). In the context of a growing plant apex, this reactiondiffusion mechanism can explain both the emergence of organs de novo as well as the thresholding mechanism of an inhibition model of phyllotaxis.

Figure 4.5 shows the activator-inhibitor model on the growing leaf model described in Section 2.3. The diffusion term in Equations 4.2 and 4.3 need only be modified to take into account the differing area of cells and lengths of cell walls. Initially, the leaf area is filled with fairly uniformly spaced peaks in activator concentration. As the leaf grows, space opens up to create room for more peaks. In this case the spacing 


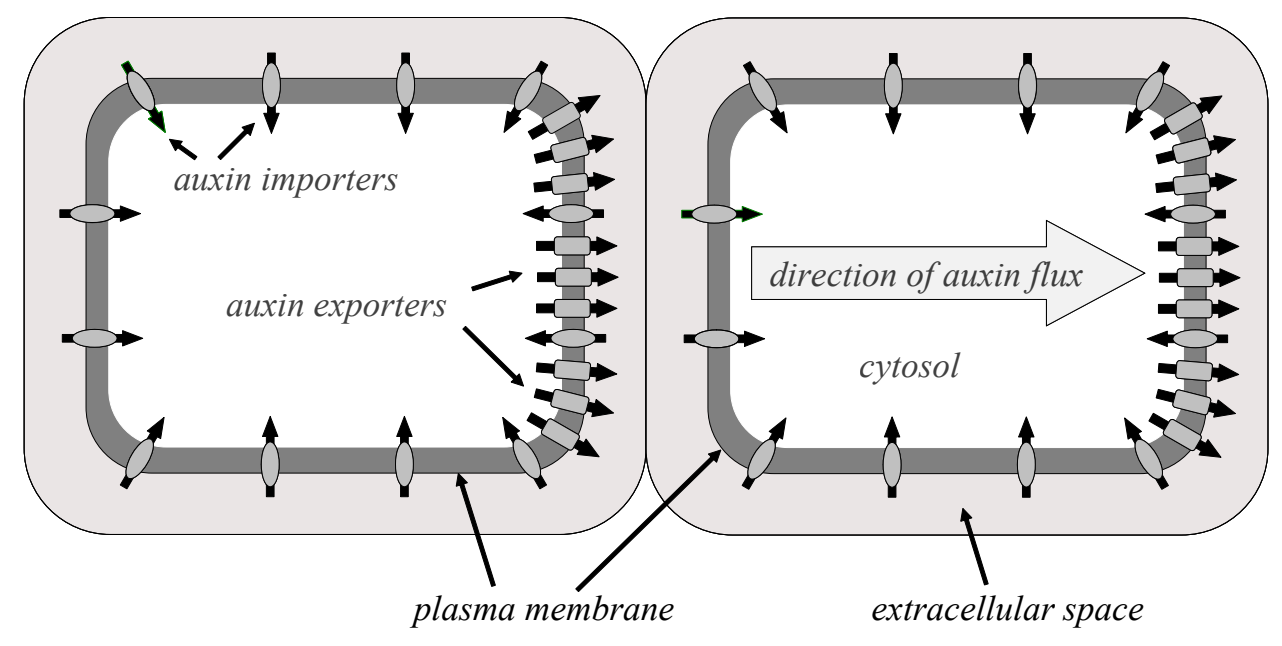

Fig. 4.6 Auxin transport in Arabidopsis cells. Export and import proteins sit in the plasma membrane and transfer auxin between the cytosol and extra-cellular space. Often, the export proteins are polarly localized, resulting in a net flux of auxin through the cells. (Adapted from Smith (2008)).

mechanism operates on a growing leaf, rather than a growing shoot tip. A similar mechanism is thought to regulate the spacing of trichomes in the leaf, however the genetic regulatory network involves at least five substances and is substantially more complex (Barbier de Reuille et al., 2007). Nevertheless, the underlying principle is the same.

\subsection{A spacing mechanism based on polar transport}

A spacing mechanism based on reaction-diffusion seems like a plausible explanation for phyllotaxis patterning in the shoot apex. The idea that existing primordia emit a diffusing inhibitor to suppress organ formation nearby is almost 100 years old, but no such inhibitors have been found. Instead the experimental evidence points to a different type of patterning mechanism, based on the active transport of the plant hormone auxin (indole-acetic-acid or IAA).

Plant tissue often displays a polarity with respect to auxin transport. When sections of tissue are excised, and alternate ends are exposed to higher levels of auxin, then auxin will move faster in one direction than the other. To explain this phenomenon, the chemiosmotic model of auxin transport was proposed (Rubery and Sheldrake, 1974). Auxin is a weak acid, and in the neutral pH inside cells it is largely dissociated. In this ionic form, auxin is hydrophilic and unable to cross the plasma membrane. In order for auxin to leave a cell, it requires the activity of export carrier proteins located at the plasma membrane. In in the lower $\mathrm{pH}$ of the extracellular space, auxin becomes protonated, and able to reenter the cell, although plants also have specialized auxin import carriers to aid this process. Often the export carriers display a polarity to one 
side of the cell (see Figure 4.6). When this polarity is coordinated at the tissue level, it results in a net directional flux of auxin through the tissue.

In the surface layer of cells in the shoot apex of Arabidopsis, the auxin export protein PIN1 localizes to one side of the cell, pumping auxin towards the sites where new primordia will appear (Reinhardt et al., 2003). Mutant Arabidopsis plants missing the auxin exporter protein PIN1 do not initiate organs in their inflorescence meristems at all, hence the name pin. However Reinhardt et al. (2003) were able to restore organ formation by applying micro-droplets of auxin directly to the peripheral zone. Based on these results they proposed a model for organ initiation in which the local activation of cells is not caused by local self enhanced production as is the case in reaction-diffusion models, but rather by the directed transport of auxin to organ initiation sites. Their model did not, however, explain what polarizes the exporter proteins. An hypothesis came from computer simulation studies (Jönsson et al., 2006; Smith et al., 2006), leading to the discovery of a possible new patterning mechanism in plants.

In these simulations studies, it was proposed that cells could sense the concentration of auxin in neighboring cells, and polarize PINs preferentially towards cells with higher auxin concentration. This feedback of auxin on its own transport has an autocatalytic component, if one cell has a slightly higher auxin concentration, then this causes the PIN1 proteins in neighboring cells to orient preferentially towards it, causing a further increase in concentration. This increase then causes even more PINs to orient towards it, causing an even higher auxin concentration. In a tissue of cells, this can result in a transport-feedback spacing mechanism similar to Meinhardt and Gierers activatorinhibitor system.

Again, a simple topology to look at this patterning mechanism is a line of cells, connected at the ends to form a ring. There is no growth, and unlike the case with the activator-inhibitor system, it is not necessary to consider production and decay of IAA and PIN1. Only the dynamics of the transport mechanism itself, the transport of IAA and the localization of PIN, are required for patterning.

Each cell stores an IAA concentration, and the orientation of a fixed amount of PIN1 export proteins, which can point towards the cell's left or right neighbors. The simulation begins with each cell containing an initial IAA concentration, which changes over time due to diffusion and PIN1-mediated transport to and from neighbor cells. PIN1 proteins preferentially locate on the membranes facing neighbor cells with higher IAA concentration according to the formula:

$$
\left[P I N_{i \rightarrow j}\right]=\left[P I N_{i}\right] \frac{\left[I A A_{j}\right]}{\sum_{k \in N_{i}}\left[I A A_{k}\right]}
$$

where $\left[P I N_{i \rightarrow j}\right]$ is the number of PIN1 proteins located in the membrane of cell $i$ facing neighboring cell $j,\left[I A A_{j}\right]$ is the concentration of IAA in neighbor cell $j,\left[P I N_{i}\right]$ is the total concentration of PIN1 proteins in cell $i$, and $N_{i}$ are the neighbors of cell $i$. The orientation of PIN1 towards neighbor cells given by Equation (4.4) is an exponential function of IAA concentration, although linear or higher powers of concentration will also produce patterning.

Active transport depends on the orientation of PIN1 proteins at the cell membranes. The effect of PIN1 on the efflux of auxin from cell $i$ to a neighboring cell $j$ is 
modeled using the formula:

$$
\text { transport }_{i \rightarrow j}=T\left[P I N_{i \rightarrow j}\right] \frac{\left[I A A_{i}\right]^{2}}{1+\kappa_{T}\left[I A A_{j}\right]^{2}}
$$

where $T$ is the transport coefficient, and $\kappa_{T}$ is the transport saturation coefficient. For a given number of PIN1 molecules near the wall separating cell $i$ from cell $j$, the flux of auxin from $i$ to $j$ is thus assumed to increase with the concentration of auxin in cell $i$, and saturate with the increasing concentration of auxin in cell $j$. Note that this is different from the formula used by Jönsson et al. (2006) in which saturation depends on the concentration of the source cell, not the destination cell. In Equation 4.5 transport is quadratic, however linear or other powers, or even exponential functions can be used.

The simulation proceeds in a sequence of time steps, with PIN1 polarity calculated at the beginning of each time step. The entire contents of PIN1 in a cell is allocated to the cell's membrane, with Equation (4.4) specifying the portioning out of available PIN1 to sections of the membrane facing each of the neighbor cells. The change in IAA concentration in each cell $i$ is modeled by the following equation:

$$
\begin{aligned}
\frac{d\left[I A A_{i}\right]}{d t}= & \text { diffusion }+ \text { transport } \\
= & \sum_{j \in N_{i}} D\left(\left[I A A_{j}\right]-\left[I A A_{i}\right]\right)+ \\
& \sum_{j \in N_{i}}\left(T\left[P I N_{j \rightarrow i}\right] \frac{\left[I A A_{j}\right]}{1+\kappa_{T}\left[I A A_{i}\right]}-T\left[P I N_{i \rightarrow j}\right] \frac{\left[I A A_{i}\right]}{1+\kappa_{T}\left[I A A_{j}\right]}\right)
\end{aligned}
$$

Diffusion is assumed to take place directly between neighboring cells as in the reaction-diffusion model discussed previously. This is also the case for transport, and the auxin transported out of a cell is deposited directly into the neighbor cell, bypassing extracellular space.

If the model is started in the absence of noise, all cells will begin with the same initial concentration, and therefore will have the same PIN1 orientation, transport, and diffusion. The concentration of IAA in all of the cells will remain equal, and no pattern will form. This is similar to what happens in reaction-diffusion systems in the absence of noise that is needed to "break symmetry". If even a small amount of noise is added to the model, for example by perturbing initial cell concentrations, a relatively evenly-spaced pattern of peaks in IAA concentration will form (Figure 4.7). Cells with small local maxima due to the initial conditions cause the PIN1 proteins in neighbor cells to be oriented preferentially towards them (very slightly) which causes the concentration of IAA at these maxima to increase. A positive feedback loop is then formed which will recruit even more PIN1 proteins over time. The depletion of IAA in the cells surrounding a peak prevents the formation of other peaks nearby.

The amplitude and spacing between these peaks can be controlled by manipulating model parameters, with decreased transport leading to fewer peaks, and increased transport leading to more peaks. A reverse relationship is observed when manipulating the diffusion coefficient as the spacing of the peaks is a balance between diffusion and 

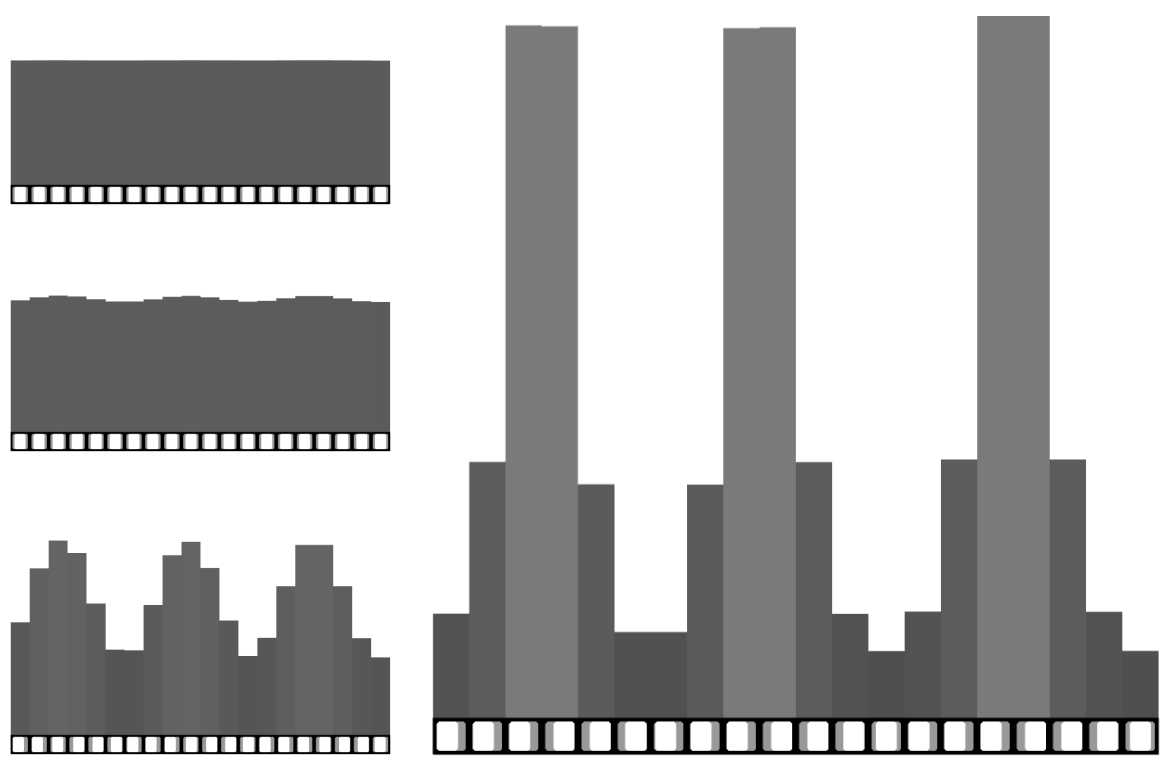

Fig. 4.7 Transport-feedback patterning on a line of 20 cells, with wrap-around boundary conditions (the leftmost and rightmost cells are considered neighbors). Taller bars (brighter) indicate higher IAA concentration. A small amount of noise present in the initial IAA distribution is required to break symmetry, and relatively evenly spaced peaks in IAA concentration are formed. Note the PIN polarization (lighter grey inside cells) towards the cells with higher auxin concentration

transport. If the diffusion coefficient is too high, or the transport coefficient is too low, no peaks will form at all. For a given cell count and parameter set, the same number of peaks usually form, regardless of the pattern of initial noise, however the locations of the peaks will vary.

\subsection{A transport-feedback model of phyllotaxis}

The transport-feedback spacing mechanism is able to make uniform peaks high in auxin concentration on a line of cells. When this model is implemented on a growing shoot apex, we would expect that as new space opens up in the center of the apex, it would be possible for new peaks to form. As in the inhibition model of phyllotaxis described in section 4.2 , the peaks would tend to form as far away from previous ones as possible, leading to phyllotaxis patterning.

In order to accommodate a growing structure, a source of auxin must be considered, as pattern formation can no longer be seen as simply redistributing auxin from some almost uniform initial state. Since it is unknown whether the auxin available in the shoot apex is supplied there by directed transport or local production, the following simplifying assumption is used. A uniform supply of auxin, modeled as local production, is provided to all cells of the apex which are outside of the central zone. These are defined to be all of the cells which are situated at a distance greater than a given 
distance from the tip of the This distance, which also corresponds to the beginning of the peripheral zone, is provided as a model parameter and remains fixed throughout the simulation.

In addition to auxin production and decay, changes must be made to equations 4.4-4.6 to reflect the different sizes of cells and cell walls. Since only the surface layer of cells is modeled, and it is assumed that all cells have the same thickness, quantities normally expressed as volumes can be expressed by areas, and areas can be expressed by lengths. This leads to the following formula for the auxin model on the cellular surface:

$$
\begin{aligned}
\frac{d\left[I A A_{i}\right]}{d t}= & \text { production }- \text { decay }+ \text { diffusion }+ \text { transport } \\
= & \frac{\rho_{I A A}}{1+\kappa_{I A A}\left[I A A_{i}\right]}-\mu_{I A A}\left[I A A_{i}\right]+\frac{D}{A_{i}} \sum_{j \in N_{i}} l_{i \rightarrow j}\left(\left[I A A_{j}\right]-\left[I A A_{i}\right]\right) \\
& \quad+\frac{T}{A_{i}} \sum_{j \in N_{i}}\left(\left[P I N_{j \rightarrow i}\right] \frac{\left[I A A_{j}\right]^{2}}{1+\kappa_{T}\left[I A A_{i}\right]^{2}}-\left[P I N_{i \rightarrow j}\right] \frac{\left[I A A_{i}\right]^{2}}{1+\kappa_{T}\left[I A A_{j}\right]^{2}}\right)
\end{aligned}
$$

where $\rho_{I A A}$ controls the production of IAA with saturation coefficient $\kappa_{I A A}$, and $\mu_{\text {IAA }}$ controls the decay of IAA which is dependent on the IAA concentration. IAA transport is modeled as in Equation 4.5 for the line model. $l_{i \rightarrow j}$ is the length of the interface between cell $i$ and cell $j$, and $A_{i}$ is the area of cell $i$. No adjustment is made for increasing cell area due to growth, or the changes in cell area that occur as a result of the pinching of cells during cell division. The formula for PIN1 localization similarly modified to account for the differing lengths of cell walls as follows:

$$
\left[P I N_{i \rightarrow j}\right]=\left[P I N_{i}\right] \frac{l_{i \rightarrow j} b^{\left[I A A_{j}\right]}}{\sum_{k \in N_{i}} l_{i \rightarrow k} b^{\left[I A A_{k}\right]}}
$$

Note that in Equations 4.7 and 4.8 the number of neighbors within the summation now varies from cell to cell. Note also that the length of cell walls does not explicitly enter the transport term in Equation 4.7, as it is already included when calculating PIN at the interface in Equation 4.8.

With equations 4.7 and 4.8, the models described in Smith et al. (2006) were able to produce reasonably spaced peaks of high auxin concentration on a growing shoot apex, however the positioning was not reliable enough to produce stable spiral phyllotaxis patterns. This was also the case for the linear equations used by Jönsson et al. (2006). Smith et al. (2006) were able to get reliable patterning by adding additional assumptions to their model that allowed cells in primordia to differentiate. These cells behaved differently from non-primordium cells and used different rules governing auxin production and PIN polarization, with PINs preferentially polarizing towards the primordium center. Although it is quite reasonable to assume that cells in newly formed primordia can differentiate, it does complicate the model somewhat. It was found that a simpler solution, the use of an exponential function for transport similar to that 

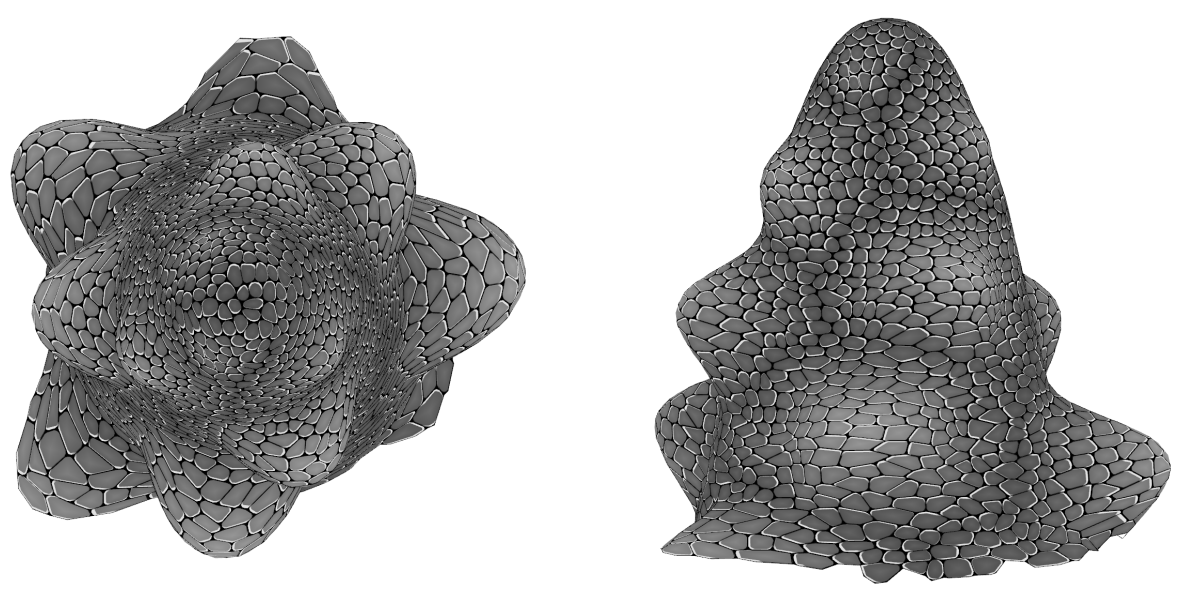

Fig. 4.8 Top (left) and side (right) views of a transport-feedback model of phyllotaxis on a growing cellular apex. Grey levels in cell interiors show auxin levels (brighter indicating more auxin) with PIN1 polarization shown in white. Convergence points of auxin and PIN1 localization appear and fill the space provided by the growing tip. The transport-feedback spacing mechanism causes new primordia to form as far as possible from previous ones, causing a spiral pattern to emerge.

used by Barbier de Reuille et al. (2006), could also produce stable patterning without any additional assumptions. In this case the transport term in equation 4.7 becomes:

$$
\text { transport }_{i \rightarrow j}=T\left[P I N_{i \rightarrow j}\right] \frac{b^{\left[I A A_{i}\right]}-1}{b^{\left[I A A_{j}\right]}}
$$

where the model parameter $b$ is the base for the exponential transport function.

Figure 4.8 shows the result of this auxin model superimposed on the growing shoot apex described in Section 2.2 with the dividing cell model from Section 2.4. As with the inhibition model, new peaks of auxin form as space appears in the peripheral zone of the growing apex tip. The auxin transport-feedback spacing mechanism causes the new primordia to form as far as possible from each other, causing a spiral pattern to emerge.

In this model the growing apex surface from Section 2.2 has been modified to allow the primordia to bulge from the surface slightly. Primordia are determined when two adjacent cells exceed a threshold concentration. The average of the cell centers is then taken as a primordium center, and projected back onto the apex surface. The radius and height increase with time, and are combined with a profile function that determines the shape of the bulging primordia. These are provided as model parameters.

\subsection{Combining molecular and physically-based models}

The phyllotaxis simulation in the previous section uses a descriptive growth model with the shape of the apex and of the outgrowing primordia specified directly in 


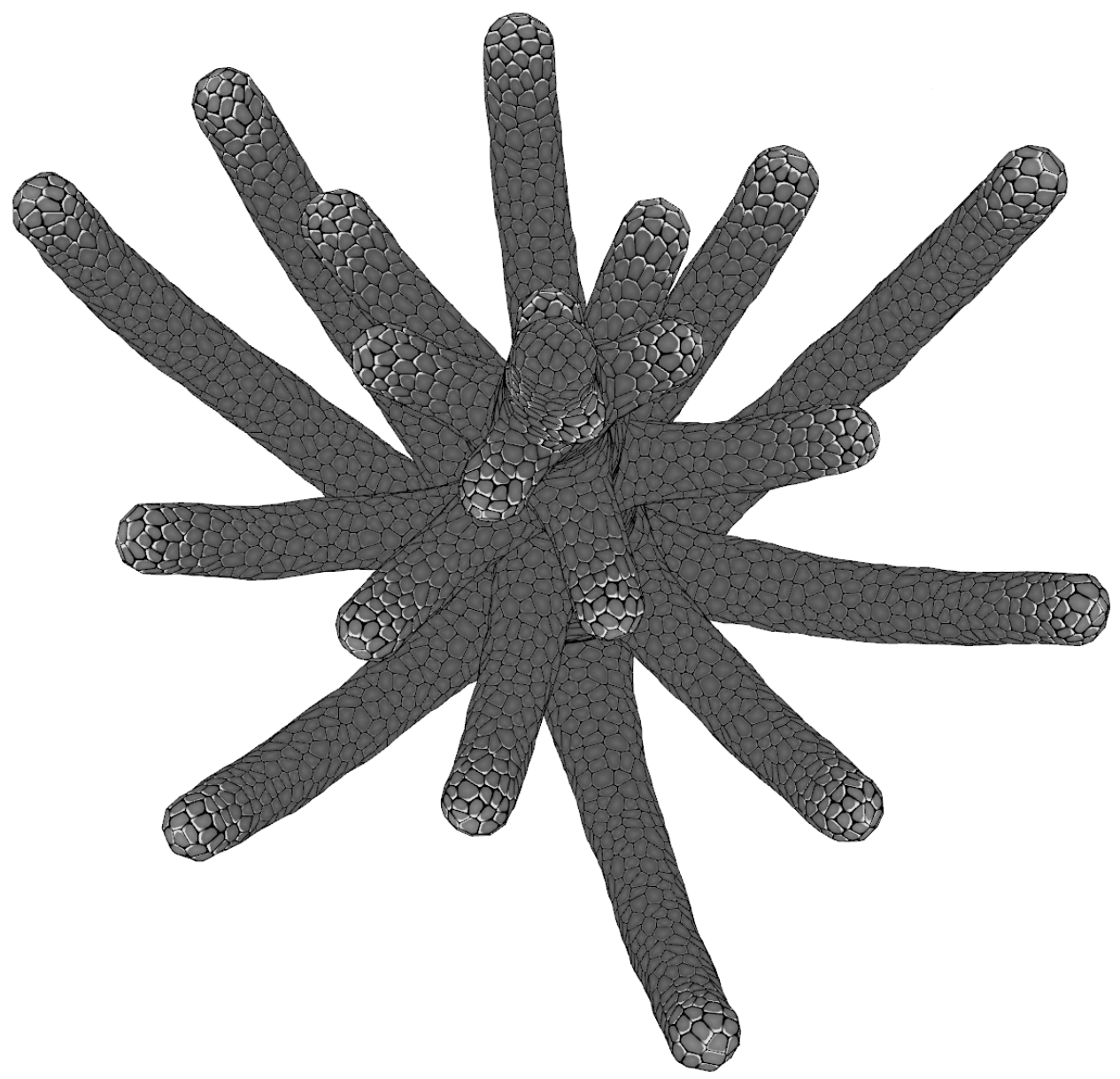

Fig. 4.9 Transport-feedback model of phyllotaxis on an apex model with physically-based (mass-spring) growth. Grey levels in cell interiors show auxin levels (brighter indicating more auxin) with PIN1 polarization shown in white. Convergence points of auxin and PIN1 localization initiate new growing tips in a spiral pattern. A slight tropism added to the primordium tip growth direction at each time step causes them to curve upwards.

geometric terms. This approach is simple and allows the modeler to focus on the patterning mechanism of phyllotaxis while abstracting from the processes involved in maintaining the shape of the apex and outgrowing primordia. With physicallybased growth models, like the one described in Chapter 3, the shape of the apex and outgrowing primordia is an emergent property of the model. Although typical more expensive computationally, this approach allows the simulation of mechanics of growth, and the interaction of biomolecules with the physical properties of cells. 
Perhaps the simplest way the molecular level phyllotaxis model and the physicallybased shoot apex model can be combined is to create new growing tips on the surface at the locations of primordia initiation. These tips are then treated in the same way as the growing apex tip, although the parameters for tip size, spring relaxation, and growth direction may be different. Figure 4.9 shows the result of such a simulation. Using the same auxin model as in the previous section, the model differs only in that auxin production is restricted to the peripheral zone, and PINs are given a preference towards the primordium center after primordium initiation. These modifications are required to prevent additional auxin peaks from forming on the flanks of primordia. In this model the primordia grow much larger, opening up space for additional auxin peaks to form.

With this model it is quite straightforward to add tropisms to developing primordia or to have lateral apices which themselves produce primordia. In addition, it is possible to model primordia which are not radially symmetric, and develop into leaves, flowers, or other organs. All of these would be much more difficult to implement in the descriptive growth models presented earlier. In addition, a physically-based model enables the possibility to study the mechanisms that determine primordium shape and extent, as well as any influence mechanics may have on primordium positioning. 


\section{Leaf venation patterning}

\subsection{Sachs' canalization hypothesis}

The intricate venation patterns seen in the leaves of vascular plants are in some cases almost as striking as spiral phyllotaxis. Equally striking, is that the same molecular components, auxin and its exporter PIN1, are the earliest known markers for vein initiation (Scarpella et al., 2006), and are key to the patterning process. However, the idea that leaf venation was a transport-feedback type of patterning process predates the discovery of the PIN1 transporter itself, although studies of the polar nature of auxin movement had predicted their existence. This lead to the chemiosmotic model of auxin transport (Rubery and Sheldrake, 1974) (see Section 4.4).

Although a reaction-diffusion model of leaf venation was proposed by Meinhardt (1982), experimental support appears to favor Sach's canalization hypothesis (Sachs, 1981). Sachs proposed that the canalization of auxin into preferred routes of auxin flux occurs by auxin feeding back on its own transport. He draws an analogy to how water
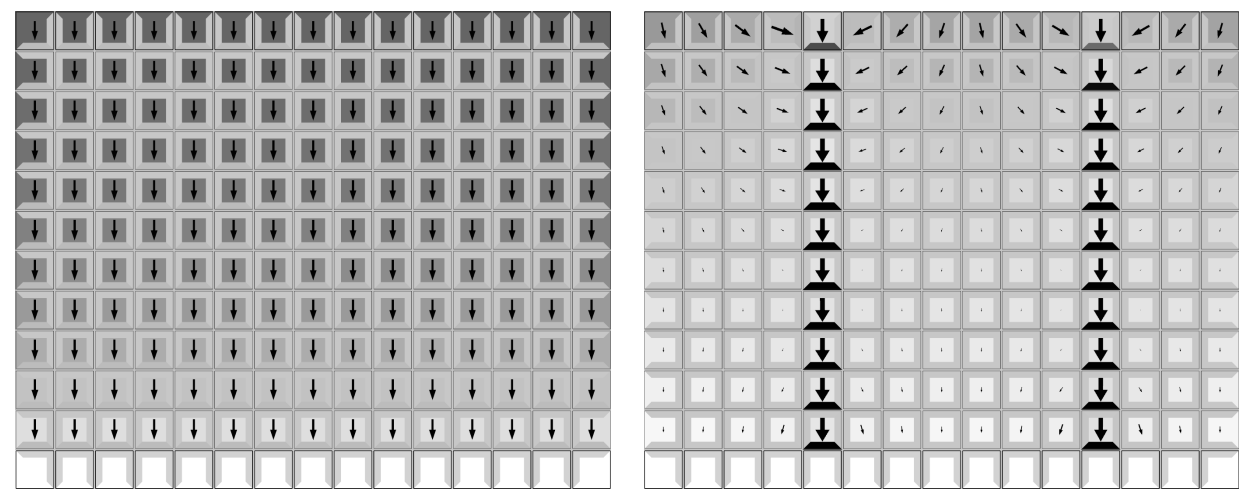

Fig. 5.1 The transport-feedback canalization mechanism for vein formation implemented on a grid of cells. Grey levels in cell interiors show auxin levels (darker indicating more auxin) with PIN1 polarization shown in the trapezoids at the cell peripheries (darker indicates more PIN1). Black arrows represent amount and principal direction of flux. Initially (left) a fairly uniform flux is present from the line of source cells (top row), to the line of sink cells (bottom row). A small amount of noise is added to the auxin concentrations, causing a destabilization and subsequent canalization into discreet auxin channels (right). Note the high flux, but low concentration in the canals. Simulation after Rolland-Lagan and Prusinkiewicz (2005). 
carves rivers in soft terrain. A cell's ability to transport auxin is assumed to increase with auxin flux, causing any initially dominant path to be reinforced. As in the case of river formation, as soon as the smallest canal begins to emerge due to random variation, it will be accentuated and attract even more flow, causing the preferred path to strengthen. Simulation models of the canalization hypothesis on grids of cells have shown that this mechanism is indeed capable of selecting strands from a tissue of undifferentiated cells (Mitchison, 1980; Rolland-Lagan and Prusinkiewicz, 2005).

Mitchison proposed two variants of the canalization model in his simulations, facilitated diffusion and polar transport, In the facilitated diffusion model, a cell's ability to transport auxin in any direction increases with flux, regardless of the direction of the flux. Transport is passive, and auxin can only move down its concentration gradient. A cell file's increased transport ability due to flux can be seen as simply increasing the rate at which auxin diffuses through and/or between cells. Mitchison's second variant, the polar transport model, is more in line with the current understanding of auxin transporters, which are thought to be highly directional. In this case, auxin exporters or carriers are allocated to sections of cell membrane facing neighbor cells based on the net flux across the cell to cell interface. The following equations from Mitchison, reformulated by Rolland-Lagan and Prusinkiewicz (2005), are used to model carrier allocation at the cell membrane. The transport or net flux $\phi_{i \rightarrow j}$ from cell $i$ to cell $j$ is given by:

$$
\phi_{i \rightarrow j}=T\left(\left[P I N_{i \rightarrow j}\right]\left[I A A_{i}\right]-\left[P I N_{j \rightarrow i}\right]\left[I A A_{j}\right]\right)+D\left(\left[I A A_{i}\right]-\left[I A A_{j}\right]\right)
$$

where $D$ is the diffusion coefficient, $T$ is the transport coefficient, $\left[P I N_{i \rightarrow j}\right]$ is the amount of carriers allocated to the section of the membrane in cell $i$ facing cell $j$, and $\left[I A A_{i}\right]$ is the concentration of auxin in cell $i$.

Carrier allocation, or the change in PIN1 at the section of the membrane in cell $i$ facing cell $j$, is given by:

$$
\begin{array}{ll}
\frac{d\left[P I N_{i \rightarrow j}\right]}{d t}=\alpha \phi_{i \rightarrow j}^{2}+\beta-\gamma\left[P I N_{i \rightarrow j}\right] & \text { if } \phi_{i \rightarrow j}>0 \\
\frac{d\left[P I N_{i \rightarrow j}\right]}{d t}=\beta-\gamma\left[P I N_{i \rightarrow j}\right] & \text { if } \phi_{i \rightarrow j} \leq 0
\end{array}
$$

where $\alpha$ controls the strength of flux driven carrier allocation, $\beta$ controls a small amount of flux independent background carrier allocation, and $\gamma$ controls decay of carriers from the cell membrane. If the net flux is zero or negative, only a background allocation and decay are considered.

The change in concentration of auxin with respect to time can now be written as:

$$
\frac{d\left[I A A_{i}\right]}{d t}=\rho-\mu\left[I A A_{i}\right]-\sum_{j \in N_{i}} \phi_{i \rightarrow j}
$$

where $\rho$ specifies the auxin production rate in cells, $\mu$ gives the auxin decay rate, and the summation is taken over all of the neighbors $N_{i}$ of cell $i$.

A simulation of this model is shown in Figure 5.1. In this simulation, $\rho$ is zero everywhere except the top row of source cells, $\mu$ is the same in all cells except the 

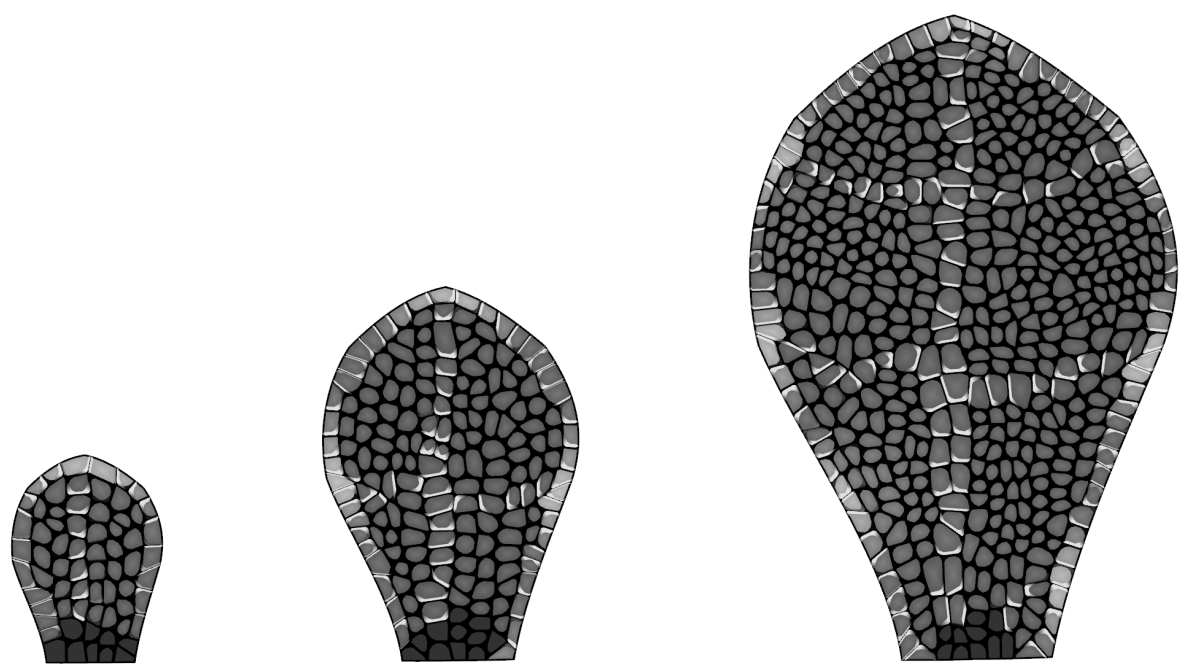

Fig. 5.2 Two transport-feedback models combined on a growing leaf surface.Grey levels in cell interiors show auxin levels (brighter indicating more auxin) with PIN1 polarization shown in white. The margin cells use the phyllotaxis mechanism (up-the-gradient) to orient the PINs, leading to the formation of peaks of auxin concentration in the margin. Upon reaching a threshold concentration, auxin begins to diffuse into the interior of the leaf, causing the peaks to act as auxin sources. The interior cells use the canalization mechanism (with-the-flux) to orient PINs, causing strands to form that connect the sources to sink cells (dark cells) at the base of the leaf. Initially (left), there is only enough margin cells for one peak to form, resulting in a single midvein on the leaf. As the leaf grows and space opens up in the margin, more peaks form, initiating secondary veins.

bottom row of sink cells, whose auxin concentration is set to zero at every time step. Initally auxin flows uniformly from the line of source cells to the line of sink cells. However, some noise in auxin levels is introduced into the model which causes some paths to be very slightly favored. The auto-catalytic reinforcement of these paths due to the non-linear production of carriers based on flux causes a destabilization into discreet canals of flux. It is these canals which are then thought go on to differentiate into veins in plant tissue.

\subsection{Combining models on a growing leaf}

The auxin and PIN1 convergence point that triggers primordium initiation in the shoot apex is thought to simultaneously trigger the formation of the midvein in the newly developing leaf. Additional convergence points in the leaf margin are then thought to initiate the first secondary veins (Scarpella et al., 2006). This suggests a simple combination of the two transport-feedback models for leaf venation and phyllotaxis. The simulation shown in Figure 5.2 uses the "up-the-gradient" PIN polarization model that makes peaks in the margin cells of the leaf, and the "with-the-flux" canalization 
model for strand formation in interior cells. The margin cells are separated from interior cells, however when their auxin concentration exceeds a threshold level, it is allowed to diffuse into the interior cells, causing veins to be initiated.

Again a small adaptation needs to be made to the canalization model equations to account for the different cell sizes. In addition, the canalization model in the previous section used a linear function of concentration for transport with no saturation, and the phyllotaxis simulations discussed previously used an exponential form with saturation. In this case it is convenient to use the same model for auxin transport for both processes, and a quadratic form is used. Thus the equations for flux in the both models become:

$$
\phi_{i \rightarrow j}=T\left[P I N_{i \rightarrow j}\right] \frac{\left[I A A_{i}\right]^{2}}{1+\kappa_{T}\left[I A A_{j}\right]^{2}}+D\left(\left[I A A_{i}\right]-\left[I A A_{j}\right]\right)
$$

with symbols as defined previously. Carrier allocation in interior cells is given by Equations 5.2 and 5.3 (with-the-flux). Carrier allocation in margin cells is given by Equation 4.8 (up-the-gradient), except we'll divide by the length of cell walls since it will be accounted for in Equation 5.6. The auxin model for both cell types then becomes:

$$
\frac{d\left[I A A_{i}\right]}{d t}=\frac{\rho_{I A A}}{1+\kappa_{I A A}\left[I A A_{i}\right]}-\mu_{I A A}\left[I A A_{i}\right]-\frac{1}{A_{i}} \sum_{j \in N_{i}} l_{i \rightarrow j} \phi_{i \rightarrow j}
$$

where the auxin production constant $\rho$ is set to zero in interior cells.

In the early stages of the simulation (Figure 5.2) there is only room in the margin for one auxin peak. After the concentration of auxin at this peak builds sufficiently, it diffuses into the interior causing the midvein to initiate, connecting the auxin source to sink cells at the base of the leaf. These sink cells represent the effect of existing vasculature of the plant. As the leaf grows, space opens up for more peaks to form, initiating secondary veins that connect to the midvein.

\subsection{A dual transport-feedback model for midvein formation}

Although the model in the previous section is able to explain the formation of veins on a growing leaf, it has several drawbacks. Mitchison's canalization model predicts high flux but low concentration in developing canals, yet experimental work indicates that the concentration of auxin is likely high (Scarpella et al., 2006). In addition, the two methods for PIN1 polarization, and thus the two patterning mechanisms, operate independently in different cells types in the previous model. However experimental work suggests that both processes must operate in both cell types (Bayer et al., 2009). In the surface layer of cells, PINs first orient towards the convergence point that marks where the new organ primordium will emerge, but then later the cells at the center of this convergence point "switch" and orient towards the interior, initiating the midvein. The same is true for cells in the inner layers. Just below the site of primordium initiation, cells first orient their PINs apically towards convergence point, but later orient downward as the convergence point extends into inner layers to form the midvein. This suggests that both cell types are able to use both methods, withthe-flux and up-the-gradient, to orient PINs. 


\section{Leaf venation patterning}

This leads to a model in which PINs can be allocated both by auxin concentration and by auxin flux (Bayer et al., 2009). The first equation of the model describes the total production and decay of PIN1 in each cell:

$$
\frac{d\left[P I N_{i}\right]}{d t}=\frac{\rho_{P I N_{0}}+\rho_{P I N}\left[I A A_{i}\right]}{1+\kappa_{P I N}\left[P I N_{i}\right]}-\mu_{P I N}\left[P I N_{i}\right]
$$

where $\rho_{P I N_{0}}$ represents PIN1 production independent of auxin levels, $\rho_{P I N}$ specifies auxin dependent PIN1 production, $\kappa_{P I N}$ is a saturation coefficient, and $\mu_{P I N}$ is the decay coefficient. As the simulation proceeds, each cell will develop a pool of PIN1 proteins available for allocation to cell membrane sections facing other cells. Since the surface layer is considered to be a different cell type then the inner tissue, the parameters for auxin dependent and independent PIN production are different in the two cell types. The surface layer of cells always expresses PINS, whereas in interior cells, PINs are only expressed in cells that are high in auxin.

In the phyllotaxis model, all PINs are allocated instantaneously at the beginning of each timestep, whereas in the canalization models, PINs accumulate at cell interfaces over time. In order to combine the two models, a variable is added $F_{i \rightarrow j}$ which records the flux history across the interface between cell $i$ and $j$. This allows the instantaneous allocation of PINs for the canalization model. The equation to calculate this flux history is defined as:

$$
\frac{d F_{i \rightarrow j}}{d t}=B_{(i, j)} \cdot \frac{\rho_{F_{0}}+\rho_{F}\left(\max \left(0, \phi_{i \rightarrow j}\right)\right)^{2}}{1+\kappa_{F} F_{i \rightarrow j}^{2}}-\mu_{F} F_{i \rightarrow j}
$$

where $\phi_{i \rightarrow j}$ is the auxin flux from cell $i$ to $j, \rho_{F_{0}}$ specifies a small default rate of increase, $\rho_{F}$ specifies the auxin flux dependent increase in flux history, $\kappa_{F}$ is a saturation coefficient, and $\mu_{F}$ specifies the rate of decay. Note that the measure of how much flux has crossed the interface recently is quadratically dependent on auxin flux, and only increases if the flux is positive. This is similar to the rule for flux-based carrier allocation in Mitchison's simulation model of canalization. The term $B_{(i, j)}$ represents the separation between the surface layer and interior cells. If the cell $i$ and $j$ are of the same type, $B_{(i, j)}=1$, otherwise it is a lesser value which is specified as a model parameter. This gives the effect of reducing communication between the surface layer and interior cells in the meristem.

The next equation describes how the choice is made between flux-based and concentrationbased PIN1 allocation. A variable $z_{i}$ is defined that is used to make this transition based on the auxin concentration of cell $i$.

$$
z_{i}=\min \left(\frac{\left[I A A_{i}\right]}{S_{P I N}}, 1.0\right)
$$

As the auxin concentration increases from 0 to a threshold concentration $S_{P I N}$, $z_{i}$ increases linearly from 0 to 1 . The variable $Z_{i \rightarrow j}$ is now defined to represent the allocation amount from the three possible sources: flux-based allocation, concentrationbased allocation, and default or background allocation.

$$
Z_{i \rightarrow j}=L_{D}+\left(1.0-z_{i}\right) L_{C}\left[I A A_{j}\right]+z_{i} L_{F} F_{i \rightarrow j}
$$


$L_{D}$ specifies a small amount of background PIN1 allocation independent of auxin flux or concentration, $L_{C}$ specifies allocation based on the concentration of neighbor cells, and $L_{F}$ specifies allocation based on flux. The total amount of PIN1 allocated to each membrane section from the pool of PIN in each cell can now be specified as:

$$
\left[P I N_{i \rightarrow j}\right]=\left[P I N_{i}\right] \frac{B_{(i, j)} \cdot l_{i \rightarrow j} b^{Z_{i \rightarrow j}}}{\sum_{k \in N_{i}} B_{(i, k)} \cdot l_{i \rightarrow k} b^{Z_{i \rightarrow k}}}
$$

where $k \in N_{i}$ are the neighbors of cell i, $\left[P I N_{i \rightarrow j}\right]$ is the amount of PIN1 on the membrane section of cell $i$ facing cell $j,\left[P I N_{i}\right]$ is the total amount of PIN1 in cell $i$, $l_{i \rightarrow j}$ is the length of the wall between cell $i$ and $j$, and $b$ is the strength of exponential allocation, given as a model parameter. Again the term $B_{(i, j)}$ reduces, but does not eliminate, allocation across the boundary between the surface layer of cells and inner layers. The complete auxin model can now be expressed as:

$$
\begin{aligned}
\frac{d\left[I A A_{i}\right]}{d t}= & \text { production }- \text { decay }+ \text { diffusion }+ \text { transport } \\
= & \frac{\rho_{I A A}}{1+\kappa_{I A A}\left[I A A_{i}\right]}-\mu_{I A A}\left[I A A_{i}\right]+\frac{D}{A_{i}} \sum_{j \in N_{i}} B_{(i, j)} l_{i \rightarrow j}\left(\left[I A A_{j}\right]-\left[I A A_{i}\right]\right) \\
& +\frac{T}{A_{i}} \sum_{j \in N_{i}} B_{(i, j)}\left(\left[P I N_{j \rightarrow i}\right] \frac{\left[I A A_{j}\right]^{2}}{1+\kappa_{T}\left[I A A_{i}\right]^{2}}-\left[P I N_{i \rightarrow j}\right] \frac{\left[I A A_{i}\right]^{2}}{1+\kappa_{T}\left[I A A_{j}\right]^{2}}\right)
\end{aligned}
$$

Note that this is almost identical to Equation 4.7, except for the term $B_{(i, j)}$ used again to reduce communication between the different cell layers. Figure 5.3 shows a simulation of Equations 5.7-5.12 on a grid of cells that is used as a two-dimensional abstraction of a longitudinal section of the shoot apex. The top row of cells is supplied with a uniform source of auxin and represents the surface layer. Initially, an auxin convergence point forms via the up-the-gradient mechanism for PIN1 polarization. As the simulation proceeds, auxin levels increase, causing a switch to flux-based polarization. This creates a strand of cells that connects the convergence point which is acting as a source of auxin, to the sink cell below. This model recapitulates several experimental observations not seen in a pure canalization model. As the midvein initiates, the concentration of auxin in the strand is always elevated compared to surrounding tissues. This is consistent with the role of auxin as a signaling molecule that is likely to instruct differentiation in a concentration based manner. Additionally, when the convergence point first appears, cells in inner tissue polarize towards it, an experimentally observed phenomena which is again difficult to reconcile with a pure canalization model. The same is true for the strong lateral polarization towards the vein center.

\subsection{A dual transport-feedback model on a growing cellular template}

A more realistic representation of the shoot apex can be made by digitizing a cross section of a real apex from confocal images (Bayer et al., 2009). In the model that 

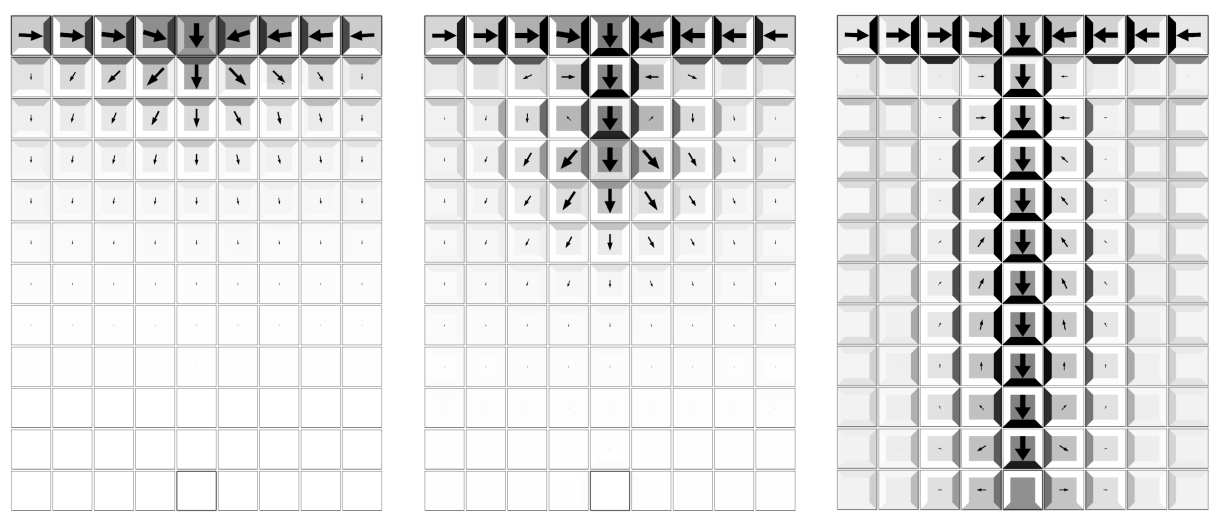

Fig. 5.3 Dual model of transport-feedback patterning on a grid of cells that represents a longitudinal section of the shoot apex. Grey levels in cell interiors show auxin levels (darker indicating more auxin) with PIN1 polarization shown in the trapezoids at the cell peripheries (darker indicates more PIN1). Black arrows represent amount and principal direction of flux. Auxin is supplied uniformly to the model in the top row of cells which represent the surface layer of the meristem. Initially (left) up-the-gradient PIN1 polarization predominates in these cells, causing a convergence point of PIN polarity and high auxin levels to emerge. Note the apical polarization in the cells in the rows below. As the simulation proceeds, auxin levels increase, and polarization switches from up-the-gradient to with-the-flux (middle), and a strand emerges (right) connecting the convergence point to the single sink cell at the bottom center of the grid. Note the high level of auxin in the emergent strand.

follows this digitization was done using the Merrysim software (Barbier de Reuille et al., 2005). Growth was added to this model by using the same Bezier surface interpolation used for the the growing leaf from Section 2.3. In this way the simulation of the formation of the midvein can include growth as the primordium bulges from the meristem surface.

When implemented on an irregular cellular surface, it was found that in the model defined by Equations 5.7-5.12, the emerging strand did not always connect directly to the sink below. Instead, the tip seemed to wander due to the irregular geometry of the cellular structure. This is not surprising since high auxin levels in the vein imply a strong auxin gradient in the proximity of the growing tip. This sharp local gradient would easily overwhelm any much shallower global gradient of auxin in the tissue leading towards the sink. It is an open question then how auxin could be both high in the extending vein, and simultaneously attract newly forming veins to each other via a "sink" effect (Heisler and Jönsson, 2006; Bayer et al., 2009; Smith and Bayer, 2009). In the model of Bayer et al. (2009) it was suggested that something else must guide veins to the sink, possibly via an innate tissue polarity, a property of plant tissue demonstrated by Sachs (1981). This polarity can be modeled in several ways, however the simplest is by simply favoring allocation to cell walls facing the sink. A small change to Equation 5.10 to add a geometrical bias $V_{i \rightarrow j}$ in the flux allocation component is made as follows: 

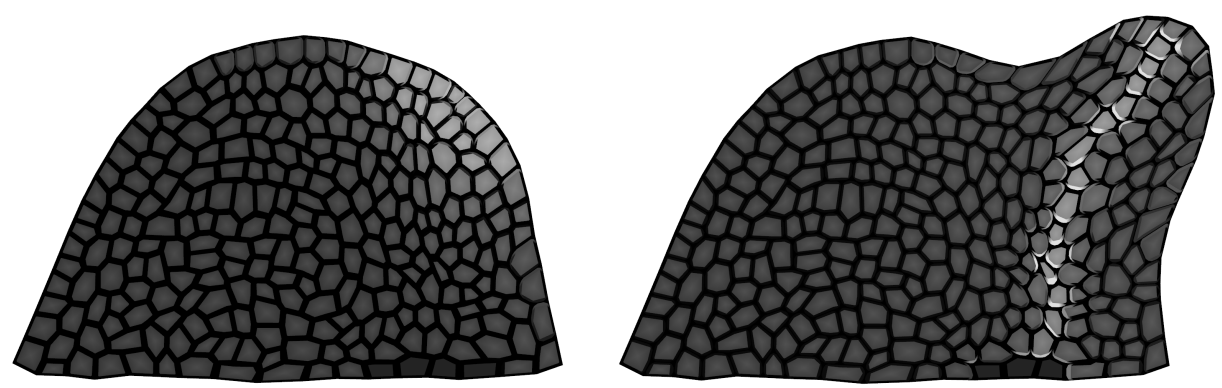

Fig. 5.4 Dual model of transport-feedback patterning on a growing cellular representation of a cross section of the shoot apex. Grey levels in cell interiors show auxin levels (lighter indicating more auxin) with PIN1 polarization shown at the cell peripheries (lighter indicates more PIN1). Auxin is supplied uniformly to the model in a range of cells in the top layer (approximately 15 cells). Initially (left) up-the-gradient PIN1 polarization causes a convergence point to form in the surface layer with apical polarization of the cells below. As the simulation proceeds, the primordium bulges from the surface as a strand emerges (right) connecting the convergence point at the center of the primordium to the sink cells (dark cells) below.

$$
Z_{i \rightarrow j}=L_{D}+\left(1.0-z_{i}\right) L_{C}\left[I A A_{j}\right]+z_{i} L_{F} V_{i \rightarrow j} F_{i \rightarrow j}
$$

where $V_{i \rightarrow j}$ is set to 0 at the location in the cell most distal from the sink cells, and set to 1 at the location nearest the sink cells. Figure 5.4 shows the results of the simulation. As in the simulation on the grid of cells, the surface layer is provided with a uniform quantity of auxin in the cells near where the primordium will form. At low auxin levels, the surface cells form a convergence point of auxin and PIN polarity. This marks the center of where the primordium will form. As auxin levels increase, the polarization mechanism switches from up-the-gradient to with-the-flux, causing a strand of cells with high auxin and PIN expression levels to appear, connecting the convergence point to the sink cells below. Note that there is almost no background production of PINs in the inner layers of cells, and that PIN expression depends almost entirely on auxin dependent production of PINs. As with the model on the rectangular grid of cells, there is strong lateral polarization of PINs towards the center of the vein, as well as high auxin levels in the developing strand. 


\section{6}

\section{Conclusion}

Perhaps the most successful formalism for modeling plant development has been Lsystems, which are ideally suited for modeling growing branching structures at the tissue level. In recent years, as our knowledge of genes, proteins, and signaling molecules has expanded, there has been an increased interest in understanding plant development at the cellular level. This is understandable, as the cell is the fundamental building block of life and it is at this level that many key developmental decisions are made. Modeling development at this level, however, has some difficult challenges. Not only is it required to develop models of genetic networks and cell to cell signaling, but these models must often be implemented on growing cellular structures in order to be relevant. Phyllotaxis patterning is a good example; without a growing structure with cells and cell division, any model proposed will always be perceived to be somewhat abstract. Except in some very special cases of filamentous organisms, most cellular structures are three dimensional, although it is often possible to abstract to two dimensions. Most of the models presented in these notes use this simplification, and the formalism of Vertex-Vertex systems (VV) for their implementation. However it is clear that methods need to be developed to conveniently represent growing, three dimensional, cellular structures.

As computational power increases, it will be possible to explore a wider range of problems, such as physically-based simulations of the growth of cellular tissues which are currently pushing the limits of technology. It is these models, however, that enable the possibility to simulate a direct link between genes, growth, and form. Genes drive the chemistry that determines the physical properties of cells, which in turn determines how cells grow. In plants, it is the control of this growth that ultimately results in the emergence of form. 


\section{7}

\section{Acknowledgments}

Support for this work was provided by the University of Bern, the Swiss SystemsX.ch project Plant Growth in a Changing Environment (PGCE), the Natural Sciences and Engineering Research Council of Canada (NSERC), the Alberta Informatics Circle of Research Excellence (iCORE), and the University of Calgary (Ruby doctoral scholarship) and is gratefully acknowledged. I would also like to acknowledge Przemyslaw Prusinkiewicz, Cris Kuhlemeier, Emmanuelle Bayer, Soazig Guyomarch, Naomi Nakayama, Pierre Barbier de Reuille, Adam Runions, and Colin Smith for insightful discussions and for helping to formulate many of the ideas appearing in the co-authored works from which a substantial portion of these notes were derived. 


\section{References}

Barbier de Reuille, Pierre, Bohn-Courseau, Isabelle, Godin, Christophe, and Traas, Jan (2005, Dec). A protocol to analyse cellular dynamics during plant development. Plant J, 44(6), 1045-1053.

Barbier de Reuille, Pierre, Bohn-Courseau, Isabelle, Ljung, Karin, Morin, Halima, Carraro, Nicola, Godin, Christophe, and Traas, Jan (2006, January). Computer simulations reveal properties of the cell-cell signaling network at the shoot apex in Arabidopsis. Proceedings of the National Academy of Science of the USA, 103(5), 1627-1632.

Barbier de Reuille, Pierre, Runions, Adam, Smith, Richard, Coen, Enrico, and Prusinkiewicz, Przemyslaw (2007). Trichome patterning on growing tissue. In Proceedings of the 5th International Workshop on Functional-Structural Plant Models, pp. 365-370.

Bayer, Emmanuelle M, Smith, Richard S, Mandel, Therese, Nakayama, Naomi, Sauer, Michael, Prusinkiewicz, Przemyslaw, and Kuhlemeier, Cris (2009, Feb). Integration of transport-based models for phyllotaxis and midvein formation. Genes Dev, 23(3), 373-384.

de Boer, M., Fracchia, F., and Prusinkiewicz, P. (1992). Lindenmayer systems: Impacts on theoretical computer science, computer graphics, and developmental biology, Chapter A model for cellular development in morphogenetic fields, pp. 351-370. Springer-Verlag, Berlin.

Dumais, Jacques (2007, Feb). Can mechanics control pattern formation in plants? Curr Opin Plant Biol, 10(1), 58-62.

Errera, L. (1888). Über zellfromen und seifenblasen. Botanisches Centralblatt, 34, 395-398.

Foley, J. D., van Dam, A., Feiner, S. K., and Hughes, J. F. (1990). Computer graphics - Principles and practice (2nd edn). Addison Wesley.

Giavitto, Jean-Louis and Michel, Olivier (2001). MGS: a ruled-based language for complex objects and collections. Electronic Notes in Theoretical Computer Science, 59(4), 1-19.

Gierer, A. and Meinhardt, H. (1972, Dec). A theory of biological pattern formation. Kybernetik, 12(1), 30-39.

Green, Paul B., Steele, C. S., and Rennich, S. C. (1996, May). Phyllotactic patterns: a biophysical mechanism for their origin. Annals of Botany, 77(5), 515-528.

Grieneisen, Verônica A, Xu, Jian, Marée, Athanasius F M, Hogeweg, Paulien, and Scheres, Ben (2007, Oct). Auxin transport is sufficient to generate a maximum and gradient guiding root growth. Nature, 449(7165), 1008-1013.

Hamant, Olivier, Heisler, Marcus G, Jnsson, Henrik, Krupinski, Pawel, Uyttewaal, Magalie, Bokov, Plamen, Corson, Francis, Sahlin, Patrik, Boudaoud, Arezki, 
Meyerowitz, Elliot M, Couder, Yves, and Traas, Jan (2008, Dec). Developmental patterning by mechanical signals in arabidopsis. Science, 322(5908), 1650-1655.

Heisler, Markus G. and Jönsson, Henrik (2006, November). Modeling auxin transport and plant development. Journal of Plant Growth Regulation, 25, 302-312.

Hejnowicz, Z., Nakielski, J., and Hejnowicz, K. (1984). Modeling of spatial variations of growth within apical domes by means of the growth tensor. ii. growth specified on dome surface. Acta Societatis Botanicorum Poloniae, 53, 301-316.

Hofmeister, Wilhelm (1868). Handbuch der Physiologishen Botanik. Engelmann, Leipzig.

Honda, Hisao (1978). Description of cellular patterns by Dirichlet domains: the twodimensional case. Journal of Theoretical Biology, 72, 523-543.

Jönsson, Henrik, Heisler, Marcus G, Shapiro, Bruce E, Meyerowitz, Elliot M, and Mjolsness, Eric (2006, Jan). An auxin-driven polarized transport model for phyllotaxis. Proc Natl Acad Sci U S A, 103(5), 1633-1638.

Kramer, Eric M (2002, May). A mathematical model of pattern formation in the vascular cambium of trees. Journal of Theoretical Biology, 216(2), 147-158.

Kramer, Eric M. (2004, December). PIN and AUX/LAX proteins: their role in auxin accumulation. Trends in Plant Science, 9(12), 578-582.

Lindenmayer, Aristid (1968a). Mathematical models for cellular interactions in development. I. Filaments with one-sided inputs. Journal of Theoretical Biology, 18(3), 280-299.

Lindenmayer, Aristid (1968b). Mathematical models for cellular interactions in development. II. Simple and branching filaments with two-sided inputs. Journal of Theoretical Biology, 18(3), 300-315.

Lindenmayer, A. and Rozenberg, G. (1979). Graph grammars and their application to computer science; First International Workshop, Lecture Notes in Computer Science 73, Chapter Parallel generation of maps: Developmental systems for cell layers, pp. 301-316. Springer-Verlag, Berlin.

Lyndon, R. F. (1998). The shoot apical meristem. Cambridge University Press.

Meinhardt, Hans (1982). Models of Biological Pattern Formation. Academic Press.

Mitchison, G. J (1980). A model for vein formation in higher plants. Philosophical

Transactions of the Royal Society of London. B. Biological Sciences, 207, 79-109.

Nakielski, Jerzy (2000). Pattern formation in biology, vision and dynamics, Chapter Tensorial model for growth and cell division in the shoot apex, pp. 252-286. World Scientific.

Neider, Jackie, Davis, Tom, and Woo, Mason (1994). OpenGL Programming Guide 9 (The Red Book). Addison-Wesley.

Prusinkiewicz, Przemyslaw (1986). Graphical applications of l-systems. In In Proceedings of Graphics Interface 86 Vision Interface 86, pp. 247-253.

Prusinkiewicz, P. (2004). Art and science for life: Designing and growing virtual plants with l-systems. Acta Hortic, 630, 1528.

Prusinkiewicz, Przemyslaw, Hammel, Mark, Hanan, Jim, and Mech, Radomr (1996). Visual models of plant development.

Prusinkiewicz, Przemyslaw and Lindenmayer, Aristid (1990). Algorithmic Beauty of Plants. Springer-Verlag. 
Reinhardt, Didier, Pesce, Eva-Rachele, Stieger, Pia, Mandel, Therese, Baltensperger, Kurt, Bennett, Malcolm, Traas, Jan, Friml, Jir, and Kuhlemeier, Cris (2003, Nov). Regulation of phyllotaxis by polar auxin transport. Nature, 426(6964), 255-260.

Rolland-Lagan, A. G. and Prusinkiewicz, P. (2005, December). Reviewing models of auxin canalization in the context of leaf vein pattern formation in Arabidopsis. The Plant Journal, 44(5), 854-865.

Rubery, P.H. and Sheldrake, A.R. (1974, June). Carrier-mediated auxin transport. Planta, 118(2), 101-121.

Sachs, T. (1981). The control of patterned differentiation of vascular tissues. Advances in Botanical Research, 9, 151-262.

Scarpella, Enrico, Marcos, Danielle, Friml, Jir, and Berleth, Thomas (2006, April). Control of leaf vascular patterning by polar auxin transport. Genes and Development, 20(8), 1015-1027.

Schoute, J. C. (1913). Beitrage zur blattstellunglehre. i. die theorie. Recueilde Travaux Botániques Néerlandais., 10, 153-339.

Smith, Colin, Prusinkiewicz, Przemyslaw, and Samavati, Faramarz (2004, September). Local specification of surface subdivision algorithms. In Applications of Graph Transformations with Industrial Relevance: Second International Workshop, AGTIVE 2003 (ed. J. L. Pfaltz, M. Nagl, and B. Blen), pp. 313-327. Springer-Verlag $\mathrm{GmbH}$.

Smith, Richard S (2008, Dec). The role of auxin transport in plant patterning mechanisms. PLoS Biol, 6(12), e323.

Smith, Richard S and Bayer, Emmanuelle M (2009, Sep). Auxin transport-feedback models of patterning in plants. Plant Cell Environ, 32(9), 1258-1271.

Smith, Richard S, Guyomarc'h, Soazig, Mandel, Therese, Reinhardt, Didier, Kuhlemeier, Cris, and Prusinkiewicz, Przemyslaw (2006, January). A plausible model of phyllotaxis. Proceedings of the National Academy of Science of the USA, 103(5), 1301-1306.

Stern, Ari and Desbrun, Mathieu (2006). Discrete geometric mechanics for variational time integrators. In Discrete Differential Geometry: An Applied Introduction, a fullday course at SIGGRAPH'O6.

Stoma, Szymon, Lucas, Mikael, Chopard, Jiérôme, Schaedel, Marianne, Traas, Jan, and Godin, Christophe (2008, Oct). Flux-based transport enhancement as a plausible unifying mechanism for auxin transport in meristem development. PLoS Comput Biol, 4(10), e1000207.

Turing, A.M. (1952). The chemical basis of morphogenesis. Philosophical Transactions of the Royal Society of London. B. Biological Sciences, 237, 37-52. 